\title{
Comprendre les obstacles et les facteurs favorables au dépistage du VIH au Canada de 2009 à 2019 : examen systématique des études
} mixtes

\author{
Claudie Laprise $^{1 *}$, Clara Bolster-Foucault ${ }^{1}$
}

\section{Résumé}

Contexte : Le dépistage du VIH est un pilier central de l'approche du Canada en matière de prévention et de traitement des infections transmissibles sexuellement et par le sang (ITSS) et un processus essentiel pour atteindre la première des cibles 90-90-90 du Programme commun des Nations Unies sur le VIH/sida (ONUSIDA). Malgré les progrès réalisés vers l'atteinte de cet objectif, de nombreux Canadiens ne connaissent toujours pas leur état sérologique et les taux de dépistage varient d'une population et d'une province ou d'un territoire à l'autre. II est essentiel de comprendre les facteurs menant au dépistage du VIH pour améliorer l'accès au dépistage du VIH et atteindre les personnes qui ne connaissent pas encore leur séropositivité.

Objectif : Examiner les obstacles et les facteurs favorables au dépistage du VIH dans les populations clés et les provinces et territoires au Canada.

Méthodes : Un examen systématique d'études mixtes publiées de 2009 à 2019 dans la littérature grise et dans la littérature révisée par les pairs a été réalisé, ce qui a permis d'identifier les études quantitatives et qualitatives sur les obstacles et les facteurs favorables au dépistage du VIH au Canada. Les études ont été sélectionnées aux fins de l'examen et les obstacles et facteurs favorables retrouvés ont été extraits. La qualité des études retenues a été évaluée et les résultats ont été résumés.

Résultats : Nous avons recensé 43 études pertinentes. L'examen a fait ressortir des obstacles communs dans les populations clés et les provinces et territoires, notamment les difficultés d'accès aux services de dépistage, la peur et la stigmatisation entourant le $\mathrm{VIH}$, la perception d'un faible risque, le manque de confidentialité pour les patients et le manque de ressources pour le dépistage. Des pratiques novatrices qui pourraient faciliter le dépistage du VIH ont été identifiées, comme de nouveaux lieux de dépistage (soins dentaires, pharmacies, unités mobiles, services d'urgence), de nouvelles modalités (dépistage buccal, counseling par les pairs) et des interventions et approches personnalisées en fonction du sexe, du genre et de l'âge. Les populations clés sont également confrontées à des obstacles socioculturels, structurels et législatifs particuliers qui défavorisent le dépistage du VIH. De nombreuses études ont fait ressortir la nécessité d'offrir une vaste gamme d'options de dépistage et d'intégrer les tests dans les pratiques de soins de santé de routine.

Conclusion : Les efforts visant à améliorer l'accès au dépistage du VIH devraient tenir compte des obstacles et des facteurs favorables au dépistage au niveau de l'individu, des fournisseurs de soins de santé et des politiques. Ils devraient également mettre l'accent sur l'accessibilité, l'inclusivité, la commodité et la confidentialité des services de dépistage. Par ailleurs, les services de dépistage doivent être adaptés aux besoins et contextes particuliers des populations clés.
Citation proposée : Laprise C, Bolster-Foucault C. Comprendre les obstacles et les facteurs favorables au dépistage du VIH au Canada de 2009 à 2019 : examen systématique des études mixtes. Relevé des maladies transmissibles au Canada 2021;47(2):117-38. https://doi.org/10.14745/ccdr.v47i02a03f

Mots-clés : VIH, obstacles, facteurs favorables, dépistage, Canada, examen systématique, études mixtes, populations clés

Page 117
Cette oeuvre est mise à la disposition selon les termes de la licence internationale Creative Commons Attribution 4.0

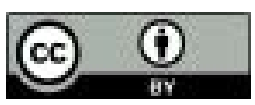

Affiliation

${ }^{1}$ Agence de la santé publique du Canada, Direction générale de l'infrastructure de sûreté sanitaire, Unité des capacités en santé publique et de la gestion du savoir, Bureau régional du Québec; Montréal, QCC

\section{*Correspondance :}

claudie.laprise@canada.ca 


\section{Introduction}

L'Organisation mondiale de la Santé a estimé qu'environ 37,9 millions de personnes vivaient avec le $\mathrm{VIH} /$ sida dans le monde en 2018, y compris 1,7 million de personnes nouvellement infectées au cours de cette année-là (1). Au Canada, plus de 63000 personnes vivaient avec le VIH en 2016, et près de 23000 nouveaux cas ont été diagnostiqués entre 2008 et 2017 (2).

Le dépistage et le diagnostic du VIH constituent une première étape cruciale dans le continuum de soins du VIH (diagnostic du VIH, aiguillage vers les soins, commencement du traitement antirétroviral et atteinte de la suppression virale). Pour les personnes vivant avec le $\mathrm{VIH}$ qui connaissent leur état sérologique, le fait de recevoir un traitement approprié réduit l'impact à long terme de la maladie et prévient la transmission (3).

En 2014, le Conseil de coordination du Programme commun des Nations Unies sur le VIH/sida (ONUSIDA) a établi les cibles 90-90-90 dans le but de mettre fin à l'épidémie du sida d'ici 2020. Ces cibles visent à ce que $90 \%$ de toutes les personnes vivant avec le $\mathrm{VIH}$ connaissent leur état sérologique, que $90 \%$ de toutes les personnes ayant reçu un diagnostic de $\mathrm{VIH}$ reçoivent un traitement antirétroviral approprié et que $90 \%$ de toutes les personnes recevant un traitement antirétroviral atteignent une suppression virale $(4,5)$. Le Canada n'a pas encore atteint le premier de ces objectifs; environ $14 \%$ des Canadiens vivant avec le VIH en 2016 n'étaient pas au courant de leur état sérologique (2).

Bien que le dépistage du VIH au Canada se fasse de plus en plus, les taux de dépistage varient considérablement entre les régions du pays (6). Les taux de dépistage régionaux peuvent être influencés par les politiques et les programmes des provinces ou territoires qui déterminent l'accessibilité des tests de dépistage et les types de tests disponibles (e.g. tests au point de service) (7). On sait également que certaines populations sont touchées de manière disproportionnée par le $\mathrm{VIH}$, notamment les hommes gais, bisexuels et autres hommes ayant des relations sexuelles avec des hommes (gbHARSAH), les personnes transgenres, les personnes qui utilisent des drogues par injection et les professionnels du sexe (8-12). En raison de la stigmatisation, de la discrimination et des déterminants sociaux de la santé, ces populations sont souvent marginalisées et mal desservies, ce qui mène à une plus grande probabilité d'acquisition et de transmission du VIH, ainsi qu'à un accès et un recours limités au dépistage $(13,14)$. La répartition différentielle de ces populations au Canada peut contribuer à la variation régionale du dépistage du $\mathrm{VIH}(2)$.

Dans le contexte canadien, il n'existe actuellement pas de vue d'ensemble exhaustive des obstacles et des facteurs favorables au dépistage du VIH dans les populations clés et dans les provinces et territoires. Ces connaissances sont essentielles pour orienter les politiques et les mesures de santé publique vers les cas non diagnostiqués et atténuer l'impact du VIH sur la santé au Canada. Deux revues de littérature décrivent les obstacles et les facteurs favorables au dépistage du VIH dans le contexte canadien $(7,15)$. Elles ont relevé de nombreux obstacles et facteurs favorables au niveau de l'individu (e.g. perception du faible risque, peur), des fournisseurs de soins de santé (e.g. contraintes de temps, ressources insuffisantes) et des institutions ou des politiques (e.g. coût, accessibilité des tests) (7,15-17). Toutefois, ces revues de littérature n'étaient pas systématiques, n'ont pas examiné la dernière décennie dans son ensemble ni les tendances du dépistage du $\mathrm{VIH}$ dans les principales populations ou dans des provinces ou territoires particuliers. De plus, peu d'études menées au Canada ont été recensées dans le cadre de ces revues.

L'objectif de cet examen systématique des études mixtes est d'examiner les obstacles et facteurs favorables au dépistage qui ont été décrits dans l'ensemble des populations et des provinces ou territoires au Canada au cours de la dernière décennie et d'effectuer une synthèse narrative des travaux recensés.

\section{Méthodes}

\section{Stratégie de recherche}

Un examen systématique des études mixtes (18) a été effectué sur les obstacles et les facteurs favorables au dépistage du VIH au Canada, conformément aux lignes directrices sur les éléments de déclaration préférés pour les examens systématiques et les méta-analyses (Preferred Reporting Items for Systematic Reviews and Meta-Analyses [PRISMA]) (19) (I'annexe sont disponibles sur demande). En se basant sur un protocole prédéfini et en collaboration avec des spécialistes de l'information, les examinateurs ont élaboré une stratégie de recherche électronique pour relever des études originales quantitatives, qualitatives, et à méthodes mixtes portant sur les obstacles et les facteurs favorables au dépistage du VIH au Canada qui ont été publiées entre le $1^{\mathrm{er}}$ janvier 2009 et le 9 décembre 2019 (l'annexe sont disponibles sur demande). Des recherches ont été menées dans Medline, Embase, Psyclnfo, ProQuest Public Health, ProQuest Sociology Collection et Scopus pour trouver des publications révisées par les pairs, ainsi que dans Google et Google Scholar pour trouver de la littérature grise, des rapports gouvernementaux et d'organisations non gouvernementales et des dissertations. Les pages Web des gouvernements de chaque province et territoire ont également été consultées, et les partenaires des bureaux régionaux de l'Agence de la santé publique du Canada ont été consultés pour repérer d'autres ouvrages pertinents. Par ailleurs, les listes de référence des études retenues ont été examinées manuellement pour trouver des publications pertinentes.

\section{Critères d'admissibilité}

Les études pouvaient être incluses s'il s'agissait d'études quantitatives ou qualitatives originales faisant état d'obstacles 
ou de facteurs favorables au dépistage du VIH dans une ou plusieurs provinces ou territoires au Canada, publiées entre le 1 janvier 2009 et le 9 décembre 2019 et rédigées en français ou en anglais. II n'y avait aucune restriction quant à la taille de l'échantillon de l'étude, au type de population à l'étude ou au contexte ou milieu de l'étude. Les études ont été exclues si on y signalait des obstacles ou des facteurs favorables au dépistage pour de multiples infections transmissibles sexuellement et par le sang (ITSS) sans déclarer les résultats du VIH séparément, ou si les données de l'étude avaient été recueillies avant 2009.

\section{Sélection des études et collecte des données}

Deux examinateurs ont passé en revue les titres de façon indépendante et les résumés de toutes les études recensées. Le texte intégral des documents potentiellement pertinents a ensuite été examiné par les deux examinateurs, de façon indépendante. Les désaccords entre les examinateurs aux étapes de la présélection et de l'examen du texte intégral ont été résolus par consensus.

Les deux examinateurs ont extrait indépendamment des données d'études incluses à l'aide d'un formulaire piloté d'extraction de données qui avait été créé à partir d'un échantillon de deux études quantitatives et de quatre études qualitatives sélectionnées en raison de leur grande qualité. Pour toutes les publications retenues, la province ou le territoire de l'étude, les buts de l'étude, le plan d'étude, la population, la taille de l'échantillon, la méthode de collecte des données, les années de collecte des données, les critères d'inclusion et d'exclusion et les données démographiques de base des participants à l'étude, notamment l'âge, le sexe ou le genre, I'orientation sexuelle, la race et l'origine ethnique ont été extraits. Pour les études quantitatives, la méthode d'analyse, l'exposition à l'étude, les résultats, les covariables et les principales mesures des effets des obstacles et des facteurs favorables relevés ont été extraits. Pour les études qualitatives, la méthode analytique et les thèmes relevés relatifs aux obstacles et aux facteurs favorables au dépistage du VIH ont été extraits.

\section{Évaluation de la qualité}

Deux chercheurs ont évalué indépendamment la qualité des travaux retenus à l'aide de l'outil d'évaluation des méthodes mixtes (Mixed Methods Appraisal Tool [MMAT]) $(20,21)$. Le MMAT a été validé pour évaluer de façon critique la qualité méthodologique des études à conceptions diverses. L'outil comprend cinq questions auxquelles il faut répondre par " Oui », par "Non » ou par " Je ne sais pas ». Les questions sont adaptées à chaque type de plan d'étude et évaluent la pertinence du plan d'étude pour la question de recherche, la probabilité de biais et la pertinence des mesures et des analyses.

En fonction des réponses à ces questions, une note de cinq points a été établie pour mesurer la qualité, un point étant attribué à chaque réponse "Oui ». Les études comportant quatre réponses « Oui » ou plus ont été jugées de qualité élevée, les études comportant trois réponses « Oui » ont été jugées de qualité modérée et les études comportant deux réponses " Oui » ou moins ont été jugées de qualité faible. Les désaccords dans la note attribuée par les deux examinateurs ont été résolus par consensus. Aucune étude n'a été exclue en raison de sa qualité, l'objectif de cet examen étant de synthétiser toutes les données disponibles sur les obstacles et les facteurs favorables au dépistage du VIH au Canada. (L'annexe sont disponibles sur demande.)

\section{Analyse des données}

Les obstacles ont été définis comme étant tout obstacle ou raison invoqué par les participants à l'étude pour refuser le dépistage du VIH ou ne pas y avoir accès. À l'inverse, les facteurs favorables au dépistage étaient définis comme toute raison donnée par les participants à l'étude pour accepter un test de dépistage du VIH ou être en mesure d'y accéder. Les caractéristiques sociodémographiques et les comportements (e.g. âge, sexe, genre, comportements sexuels) qui étaient associés à une diminution ou à une augmentation du recours au dépistage du VIH étaient considérés comme des obstacles et des facteurs favorables, respectivement. Pour éviter les répétitions, les caractéristiques sociodémographiques qui agissent à la fois comme obstacles et comme facteurs favorables au dépistage du $\mathrm{VIH}$ sont présentées en termes de caractéristiques associées à l'augmentation du recours au dépistage.

Les obstacles et les facteurs favorables au dépistage du VIH ont été analysés au moyen d'une synthèse qualitative convergente dans laquelle les données quantitatives sont transformées en résultats qualitatifs $(18,22)$. Les résultats ont ensuite été intégrés à l'aide d'une synthèse thématique inductive dans laquelle les thèmes sont dérivés des données sans cadre de codage prédéfini. La synthèse a été guidée par un cadre conceptuel élaboré par Deblonde et al. (2010) (17) qui classe les déterminants du dépistage du VIH selon le niveau auquel ils se produisent : le niveau personnel; le niveau des fournisseurs de soins de santé; et le niveau institutionnel ou des politiques. Pour atteindre les objectifs de recherche, une synthèse globale des résultats a été effectuée, suivie d'une synthèse par population clé et par province ou territoire.

\section{Résultats}

\section{Sélection et caractéristiques des études}

La recherche initiale a donné lieu à 1694 études révisées par les pairs et 49 textes dans la littérature grise. Après le retrait des doublons et des publications qui ne répondaient pas aux critères d'admissibilité en fonction de leur titre ou de leur résumé, 156 a ont été conservés pour un examen du texte intégral. De ce nombre, 33 études révisées par les pairs (23-55) et 10 textes de la littérature grise $(6,56-64)$ ont été conservés (figure 1).

Le tableau 1 présente les caractéristiques des études incluses. Les études incluses ont été menées en Colombie-Britannique ( $n=12)(23,24,26,27,30,32,34,37,38,43,50,52)$; au Manitoba 
Figure 1 : Diagramme de flux de données PRISMA

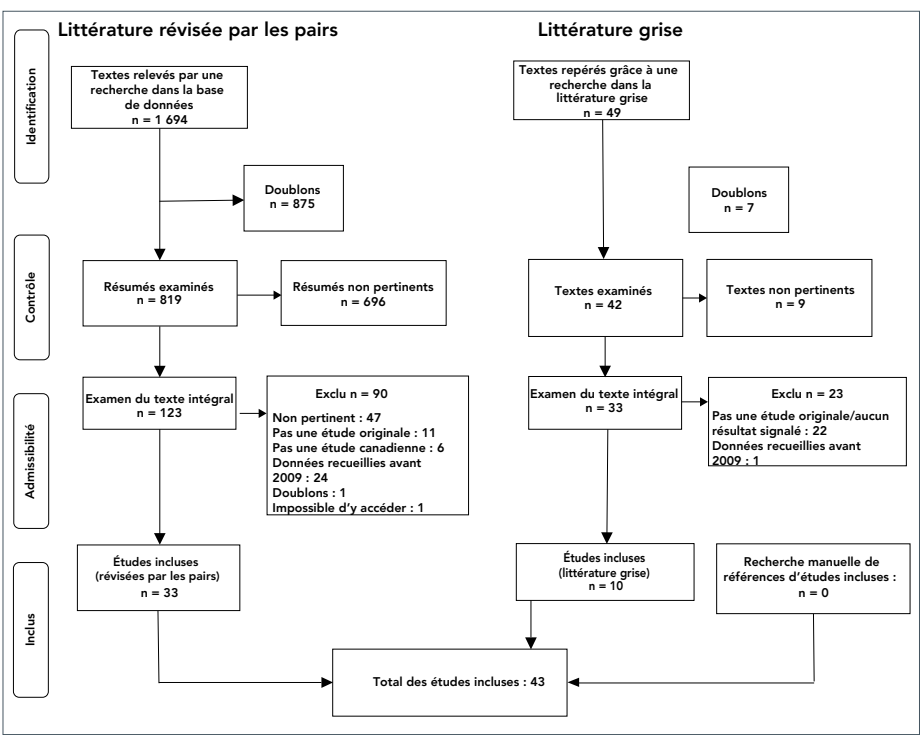

$(n=1)$ (39); en Ontario $(n=10)(35,36,40,44-47,51,60,64) ;$ au Québec $(n=5)(29,41,49,58,61)$; en Nouvelle-Écosse $(n=4)$ $(31,42,56,59)$; et à Terre-Neuve-et-Labrador $(n=1)(25)$. Sept études comprenaient plusieurs provinces et territoires (provinces de l'Atlantique $(28,62)$, ensemble du Canada $(6,33,48,54,57))$ et deux n'ont pas précisé de province ou de territoire $(53,55)$. Des 43 publications, 42 étaient des études transversales et une était une étude de cohorte. De ce nombre, 20 étaient des études quantitatives, 13 étaient des études qualitatives et 10 étaient des études à méthodes mixtes.

\section{Évaluation de la qualité}

La plupart des publications incluses étaient de bonne qualité ( $n=32 ; 74 \%)$, tandis que certaines étaient de qualité moyenne ( $n=6 ; 14 \%$ ) ou faible $(n=5 ; 12 \%)$. (L'annexe sont disponibles sur demande). L'élément le plus faible des études qualitatives était le manque de détails nécessaires pour évaluer si les données corroboraient l'interprétation des résultats. L'élément le plus faible des études quantitatives était le risque de biais

Tableau 1 : Résumé des études incluses faisant état des obstacles et des facteurs favorables au dépistage du VIH au Canada, 2009 à 2019

\begin{tabular}{|c|c|c|c|c|c|c|c|c|}
\hline $\begin{array}{l}\text { Citation et } \\
\text { emplacement }\end{array}$ & $\begin{array}{l}\text { Années de } \\
\text { collecte } \\
\text { des } \\
\text { données }\end{array}$ & $\begin{array}{l}\text { Population à } \\
\text { I'étude }\end{array}$ & $\begin{array}{c}\text { Taille de } \\
\text { l'échantillon }\end{array}$ & $\begin{array}{l}\text { Âge (en } \\
\text { années) }\end{array}$ & $\begin{array}{l}\text { Hommes } \\
(\%)\end{array}$ & Type d'étude & $\begin{array}{l}\text { Question de } \\
\text { recherche }\end{array}$ & $\begin{array}{c}\text { Note de } \\
\text { qualité } \\
(/ 5)\end{array}$ \\
\hline \multicolumn{9}{|c|}{ Littérature révisée par les pairs } \\
\hline $\begin{array}{l}\text { Anderson et al., } \\
2016 \text { (23) } \\
\text { Vancouver } \\
\text { (Colombie- } \\
\text { Britannique) }\end{array}$ & $2011-2014$ & $\begin{array}{l}\text { Travailleurs du } \\
\text { sexe migrants, } \\
\text { gestionnaires et } \\
\text { propriétaires de } \\
\text { lieux de travail } \\
\text { du sexe en } \\
\text { intérieur }\end{array}$ & 46 & $\begin{array}{l}\text { Médiane : } 42 \\
(\text { El : 24-54) }\end{array}$ & 2 & $\begin{array}{l}\text { Qualitative : } \\
\text { Entrevues semi- } \\
\text { structurées avec } \\
\text { analyse thématique }\end{array}$ & $\begin{array}{l}\text { Évaluer l'impact de } \\
\text { la criminalisation du } \\
\text { travail du sexe sur la } \\
\text { prévention du VIH et } \\
\text { des ITS }\end{array}$ & 5 \\
\hline $\begin{array}{l}\text { Armstrong et al., } \\
2019(24) \\
\text { Vancouver } \\
\text { (Colombie- } \\
\text { Britannique) }\end{array}$ & 2012-2014 & gbHARSAH & 535 & $\begin{array}{l}\text { Médiane : } 30 \\
\text { (El : 24-39) }\end{array}$ & 100 & $\begin{array}{l}\text { Quantitative : } \\
\text { Questionnaire } \\
\text { (autoadministré) }\end{array}$ & $\begin{array}{l}\text { Déterminer les } \\
\text { raisons de subir un } \\
\text { test de dépistage du } \\
\text { VIH et de ne jamais } \\
\text { avoir subi un test de } \\
\text { dépistage, et explorer } \\
\text { les corrélations du } \\
\text { dépistage }\end{array}$ & 4 \\
\hline $\begin{array}{l}\text { Boyd et al., } 2019 \\
(25) \\
\text { Terre-Neuve-et- } \\
\text { Labrador }\end{array}$ & $2006-2016$ & $\begin{array}{l}\text { Patients } \\
\text { ayant reçu un } \\
\text { diagnostic du } \\
\text { VIH }\end{array}$ & $\begin{array}{l}\text { Quantitative: } \\
58 \\
\text { Qualitative: } 10\end{array}$ & $\begin{array}{l}\text { Catégorie : } \\
20-29 \\
(20,7 \%), \\
30-39 \\
(19,0 \%), \\
40-49 \\
(41,4 \%), \\
\text { Plus de } 50 \\
(19,0 \%)\end{array}$ & 91,4 & $\begin{array}{l}\text { Méthodes mixtes: } \\
\text { Entrevues semi- } \\
\text { structurées avec } \\
\text { analyse thématique } \\
\text { et examen } \\
\text { rétrospectif des } \\
\text { textes }\end{array}$ & $\begin{array}{l}\text { Déterminer la rapidité } \\
\text { du dépistage du } \\
\text { VIH, les occasions } \\
\text { manquées de } \\
\text { dépistage et } \\
\text { les obstacles au } \\
\text { dépistage du VIH }\end{array}$ & 4 \\
\hline $\begin{array}{l}\text { Brondani et al., } \\
2016 \text { (26) } \\
\text { Vancouver } \\
\text { (Colombie- } \\
\text { Britannique) }\end{array}$ & $2010-2015$ & $\begin{array}{l}\text { Population } \\
\text { générale }\end{array}$ & 519 & $\begin{array}{l}\text { Catégorie : } \\
19-24 \\
(15 \%), \\
25-44 \\
(74 \%), \\
\text { Plus de } 45 \\
(11 \%)\end{array}$ & 71,3 & $\begin{array}{l}\text { Quantitative: } \\
\text { Questionnaire } \\
\text { (autoadministré) }\end{array}$ & $\begin{array}{l}\text { Déterminer la réaction } \\
\text { et les attitudes des } \\
\text { patients face au } \\
\text { dépistage rapide du } \\
\text { VIH dans un contexte } \\
\text { dentaire }\end{array}$ & 3 \\
\hline $\begin{array}{l}\text { Deering et al., } \\
2015 \text { (27) } \\
\text { Vancouver } \\
\text { (Colombie- } \\
\text { Britannique) }\end{array}$ & $2010-2012$ & $\begin{array}{l}\text { Travailleuses du } \\
\text { sexe }\end{array}$ & 435 & $\begin{array}{l}\text { Médiane : } 35 \\
\text { (El : 38-42) }\end{array}$ & 0 & $\begin{array}{l}\text { Quantitative: } \\
\text { Questionnaire } \\
\text { (administré par } \\
\text { l'intervieweur) }\end{array}$ & $\begin{array}{l}\text { Évaluer la prévalence } \\
\text { et les corrélations de } \\
\text { l'accès au dépistage } \\
\text { du VIH }\end{array}$ & 5 \\
\hline
\end{tabular}


Tableau 1 : Résumé des études incluses faisant état des obstacles et des facteurs favorables au dépistage du VIH au Canada, 2009 à 2019 (suite)

\begin{tabular}{|c|c|c|c|c|c|c|c|c|}
\hline $\begin{array}{l}\text { Citation et } \\
\text { emplacement }\end{array}$ & $\begin{array}{l}\text { Années de } \\
\text { collecte } \\
\text { des } \\
\text { données }\end{array}$ & $\begin{array}{c}\text { Population à } \\
\text { l'étude }\end{array}$ & $\begin{array}{c}\text { Taille de } \\
\text { l'échantillon }\end{array}$ & $\begin{array}{l}\text { Âge (en } \\
\text { années) }\end{array}$ & $\begin{array}{c}\text { Hommes } \\
(\%)\end{array}$ & Type d'étude & $\begin{array}{l}\text { Question de } \\
\text { recherche }\end{array}$ & $\begin{array}{c}\text { Note de } \\
\text { qualité } \\
\text { (/5) }\end{array}$ \\
\hline
\end{tabular}

\section{Littérature révisée par les pairs (suite)}

\begin{tabular}{|c|c|c|c|c|c|c|c|c|}
\hline $\begin{array}{l}\text { Dube et al., } \\
2017 \text { (28) } \\
\text { Provinces de } \\
\text { l'Atlantique }\end{array}$ & Non indiqué & $\begin{array}{l}\text { Intervenants, } \\
\text { notamment les } \\
\text { décideurs, les } \\
\text { fournisseurs de } \\
\text { soins de santé et } \\
\text { les jeunes }\end{array}$ & 68 & Non indiqué & $\begin{array}{l}\text { Non } \\
\text { indiqué }\end{array}$ & $\begin{array}{l}\text { Qualitative : } \\
\text { Entrevues semi- } \\
\text { structurées et } \\
\text { groupes de } \\
\text { discussion avec } \\
\text { analyse thématique }\end{array}$ & $\begin{array}{l}\text { Explorer la portée } \\
\text { et l'accessibilité des } \\
\text { mesures existantes de } \\
\text { prévention du VIH et } \\
\text { du virus de l'hépatite } \\
\text { C axées sur les jeunes }\end{array}$ & 5 \\
\hline $\begin{array}{l}\text { Engler et al., } \\
2016 \text { (29) } \\
\text { Montréal } \\
\text { (Québec) }\end{array}$ & 2012-2013 & $\begin{array}{l}\text { Clients } \\
\text { hétérosexuels } \\
\text { d'une clinique } \\
\text { axée sur les } \\
\text { HARSAH }\end{array}$ & 202 & Non indiqué & 72,8 & $\begin{array}{l}\text { Quantitative : } \\
\text { Questionnaire } \\
\text { (autoadministré) }\end{array}$ & $\begin{array}{l}\text { Comprendre les } \\
\text { besoins en services } \\
\text { de prévention du } \\
\text { VIH et de santé } \\
\text { sexuelle des clientes } \\
\text { hétérosexuelles d'une } \\
\text { clinique axée sur les } \\
\text { HARSAH }\end{array}$ & 3 \\
\hline $\begin{array}{l}\text { Feng et al., } 2018 \\
\text { (30) } \\
\text { Vancouver } \\
\text { (Colombie- } \\
\text { Britannique) }\end{array}$ & 2015-2016 & $\begin{array}{l}\text { Population } \\
\text { générale }\end{array}$ & 114 & Non indiqué & 31,2 & $\begin{array}{l}\text { Méthodes } \\
\text { mixtes: Groupes } \\
\text { de discussion } \\
\text { et entrevues } \\
\text { individuelles, et } \\
\text { questionnaire } \\
\text { (autoadministré) }\end{array}$ & $\begin{array}{l}\text { Déterminer la } \\
\text { faisabilité et } \\
\text { l'acceptabilité } \\
\text { du dépistage } \\
\text { du VIH au point } \\
\text { d'intervention dans } \\
\text { les établissements } \\
\text { d'hygiène dentaire }\end{array}$ & 4 \\
\hline $\begin{array}{l}\text { Gahagan et al., } \\
2011 \text { (31) } \\
\text { Nouvelle-Écosse }\end{array}$ & 2009-2010 & $\begin{array}{l}\text { Population } \\
\text { générale }\end{array}$ & $\begin{array}{l}\text { Quantitative: } \\
15518 \\
\text { Qualitative: } 50\end{array}$ & Non indiqué & 38 & $\begin{array}{l}\text { Méthodes mixtes : } \\
\text { Entrevues semi- } \\
\text { structurées avec } \\
\text { analyse thématique } \\
\text { et données de } \\
\text { surveillance } \\
\text { régionale du VIH } \\
\text { en laboratoire }\end{array}$ & $\begin{array}{l}\text { Explorer les obstacles } \\
\text { individuels et } \\
\text { structurels et les } \\
\text { facteurs facilitant } \\
\text { les conseils et le } \\
\text { dépistage du VIH }\end{array}$ & 4 \\
\hline $\begin{array}{l}\text { Gilbert et al., } \\
2013(1,32) \\
\text { Toutes les } \\
\text { provinces }\end{array}$ & 2006-2012 & HARSAH & Non indiqué & Non indiqué & 100 & $\begin{array}{l}\text { Quantitative : } \\
\text { Données de } \\
\text { surveillance des } \\
\text { laboratoires de } \\
\text { dépistage du VIH }\end{array}$ & $\begin{array}{l}\text { Examiner l'impact du } \\
\text { dépistage du TAN } \\
\text { et de la campagne } \\
\text { de marketing social } \\
\text { sur le diagnostic de } \\
\text { l'infection aiguë au } \\
\text { VIH chez les HARSAH }\end{array}$ & 2 \\
\hline $\begin{array}{l}\text { Gilbert et al., } \\
2013(2,33) \\
\text { Toutes les } \\
\text { provinces }\end{array}$ & 2011-2012 & HARSAH & 8388 & $\begin{array}{l}\text { Médiane : } 43 \\
\text { (El : 18-84) }\end{array}$ & 100 & $\begin{array}{l}\text { Quantitative : } \\
\text { Questionnaire } \\
\text { (autoadministré) }\end{array}$ & $\begin{array}{l}\text { Évaluer les avantages } \\
\text { et les inconvénients } \\
\text { perçus des tests sur } \\
\text { Internet chez les } \\
\text { HARSAH }\end{array}$ & 5 \\
\hline $\begin{array}{l}\text { Holtzman et al., } \\
2016 \text { (34) } \\
\text { Vancouver } \\
\text { (Colombie- } \\
\text { Britannique) }\end{array}$ & 2010-2011 & $\begin{array}{l}\text { HARSAH vivant } \\
\text { à l'extérieur des } \\
\text { grands centres } \\
\text { urbains }\end{array}$ & 153 & $\begin{array}{l}\text { Moyenne : } \\
39,7 \\
(E T: 15,4)\end{array}$ & 100 & $\begin{array}{l}\text { Quantitative : } \\
\text { Questionnaire } \\
\text { (autoadministré) }\end{array}$ & $\begin{array}{l}\text { Étudier les } \\
\text { comportements et } \\
\text { les prédicteurs du } \\
\text { dépistage du VIH chez } \\
\text { les HARSAH vivant à } \\
\text { l'extérieur des grands } \\
\text { centres urbains }\end{array}$ & 5 \\
\hline $\begin{array}{l}\text { lqbal et al., } 2014 \\
\text { (35) } \\
\text { Ontario }\end{array}$ & 2011 & $\begin{array}{l}\text { Femmes } \\
\text { en travail } \\
\text { d'accouchement }\end{array}$ & 92 & $\begin{array}{l}\text { Moyenne : } 32 \\
(E T: 4,4)\end{array}$ & 0 & $\begin{array}{l}\text { Quantitative : } \\
\text { Questionnaire } \\
\text { (autoadministré) }\end{array}$ & $\begin{array}{l}\text { Évaluer les attitudes et } \\
\text { les opinions entourant } \\
\text { le dépistage du VIH au } \\
\text { point d'intervention }\end{array}$ & 2 \\
\hline $\begin{array}{l}\text { Kesler et al., } \\
2018(36) \\
\text { Toronto } \\
\text { (Ontario) }\end{array}$ & 2010-2012 & HARSAH & 150 & $\begin{array}{l}\text { Médiane : } \\
44,5 \\
(E I: 37-50)\end{array}$ & 100 & $\begin{array}{l}\text { Quantitative : } \\
\text { Questionnaire } \\
\text { (autoadministré) }\end{array}$ & $\begin{array}{l}\text { Quantifier l'impact } \\
\text { potentiel des } \\
\text { poursuites pour non- } \\
\text { divulgation sur le } \\
\text { dépistage du VIH et la } \\
\text { transmission parmi les } \\
\text { HARSAH }\end{array}$ & 4 \\
\hline
\end{tabular}


Tableau 1 : Résumé des études incluses faisant état des obstacles et des facteurs favorables au dépistage du VIH au Canada, 2009 à 2019 (suite)

\begin{tabular}{c|c|c|c|c|c|c|c|c}
$\begin{array}{c}\text { Citation et } \\
\text { emplacement }\end{array}$ & $\begin{array}{c}\text { Années de } \\
\text { collecte } \\
\text { des } \\
\text { données }\end{array}$ & $\begin{array}{c}\text { Population à } \\
\text { l'étude }\end{array}$ & $\begin{array}{c}\text { Taille de } \\
\text { l'échantillon }\end{array}$ & $\begin{array}{c}\text { Âge (en } \\
\text { années) }\end{array}$ & $\begin{array}{c}\text { Hommes } \\
(\%)\end{array}$ & Type d'étude & $\begin{array}{c}\text { Question de } \\
\text { recherche }\end{array}$ & $\begin{array}{c}\text { Note de } \\
\text { qualité } \\
(/ 5)\end{array}$
\end{tabular}

Littérature révisée par les pairs (suite)

\begin{tabular}{|c|c|c|c|c|c|c|c|c|}
\hline $\begin{array}{l}\text { Knight et al., } \\
2016(1,37) \\
\text { Vancouver } \\
\text { (Colombie- } \\
\text { Britannique) }\end{array}$ & 2013 & Jeunes hommes & 50 & $\begin{array}{l}\text { Moyenne : } \\
21,7 \\
\text { (ET : Non } \\
\text { indiqué) }\end{array}$ & 100 & $\begin{array}{l}\text { Qualitative : } \\
\text { Entrevues semi- } \\
\text { structurées avec } \\
\text { analyse critique du } \\
\text { discours }\end{array}$ & $\begin{array}{l}\text { Explorer les valeurs } \\
\text { qui influencent } \\
\text { les décisions et } \\
\text { les motivations } \\
\text { pour accéder } \\
\text { volontairement au } \\
\text { dépistage du VIH }\end{array}$ & 4 \\
\hline $\begin{array}{l}\text { Knight et al., } \\
2016(2,38) \\
\text { Vancouver } \\
\text { (Colombie- } \\
\text { Britannique) }\end{array}$ & 2013 & Jeunes hommes & 50 & $\begin{array}{l}\text { Non indiqué } \\
\text { Présumé } \\
\text { identique à } \\
\text { l'étude de } \\
\text { Knight et al," } \\
2016 \text { (37) }\end{array}$ & 100 & $\begin{array}{l}\text { Qualitative : } \\
\text { Entrevues semi- } \\
\text { structurées avec } \\
\text { analyse théorique } \\
\text { fondée }\end{array}$ & $\begin{array}{l}\text { Déterminer comment } \\
\text { la stigmatisation } \\
\text { liée au VIH est } \\
\text { ressentie de manière } \\
\text { différentielle parmi } \\
\text { les sous-groupes de } \\
\text { jeunes hommes dans } \\
\text { le cadre des pratiques } \\
\text { de dépistage } \\
\text { volontaires et de base }\end{array}$ & 5 \\
\hline $\begin{array}{l}\text { Lau et al., } 2017 \\
(39) \\
\text { Winnipeg } \\
\text { (Manitoba) }\end{array}$ & 2016 & $\begin{array}{l}\text { Patients } \\
\text { hospitalisés }\end{array}$ & 144 & $\begin{array}{l}\text { Médiane : } 58 \\
\text { (El : 42-68) }\end{array}$ & 48 & $\begin{array}{l}\text { Quantitative : } \\
\text { Questionnaire } \\
\text { (administré par } \\
\text { l'intervieweur) }\end{array}$ & $\begin{array}{l}\text { Évaluer les attitudes à } \\
\text { l'égard du dépistage } \\
\text { du VIH au point } \\
\text { de service de base } \\
\text { chez les patients } \\
\text { hospitalisés }\end{array}$ & 3 \\
\hline $\begin{array}{l}\text { Lazarus et al., } \\
2016(40) \\
\text { Ottawa (Ontario) }\end{array}$ & 2013 & $\begin{array}{l}\text { Personnes qui } \\
\text { utilisent des } \\
\text { drogues par } \\
\text { injection }\end{array}$ & 550 & $\begin{array}{l}\text { Médiane : } 43 \\
\text { (El 34-50), } \\
\text { No : 39 } \\
(\text { El : 30-48) }\end{array}$ & 78,2 & $\begin{array}{l}\text { Quantitative : } \\
\text { Questionnaire } \\
\text { (administré par } \\
\text { l'intervieweur) }\end{array}$ & $\begin{array}{l}\text { Déterminer les } \\
\text { facteurs associés au } \\
\text { recours aux tests } \\
\text { communautaires de } \\
\text { dépistage du VIH au } \\
\text { point de service }\end{array}$ & 4 \\
\hline $\begin{array}{l}\text { Lessard et al., } \\
2015 \text { (41) } \\
\text { Montréal, } \\
\text { (Québec) }\end{array}$ & 2013-2014 & $\begin{array}{l}\text { HARSAH } \\
\text { immigrants }\end{array}$ & 40 & $\begin{array}{l}\text { Moyenne : } 33 \\
(\text { ET : 10) }\end{array}$ & 100 & $\begin{array}{l}\text { Méthodes } \\
\text { mixtes : Entretien } \\
\text { téléphonique avec } \\
\text { analyse thématique }\end{array}$ & $\begin{array}{l}\text { Analyser les facteurs } \\
\text { qui contribuent } \\
\text { au recours par les } \\
\text { HARSAH immigrants } \\
\text { d'un dépistage } \\
\text { rapide du VIH dans la } \\
\text { collectivité }\end{array}$ & 3 \\
\hline $\begin{array}{l}\text { Lewis et al., } \\
2013(42) \\
\text { Halifax } \\
\text { (Nouvelle- } \\
\text { Écosse) }\end{array}$ & 2011 & $\begin{array}{l}\text { Population } \\
\text { générale }\end{array}$ & 258 & $78,1 \% 20-40$ & 53,5 & $\begin{array}{l}\text { Quantitative : } \\
\text { Questionnaire } \\
\text { (autoadministré) }\end{array}$ & $\begin{array}{l}\text { Évaluer la demande } \\
\text { communautaire pour } \\
\text { un dépistage rapide } \\
\text { du VIH au point } \\
\text { d'intervention }\end{array}$ & 4 \\
\hline $\begin{array}{l}\text { Markwick et al., } \\
2014 \text { (43) } \\
\text { Vancouver } \\
\text { (Colombie- } \\
\text { Britannique) }\end{array}$ & 2011-2012 & $\begin{array}{l}\text { Personnes qui } \\
\text { utilisent des } \\
\text { drogues par } \\
\text { injection }\end{array}$ & 600 & $50,8 \%>48$ & 67,5 & $\begin{array}{l}\text { Quantitative : } \\
\text { Questionnaire } \\
\text { (administré par } \\
\text { l'intervieweur) }\end{array}$ & $\begin{array}{l}\text { Caractériser la volonté } \\
\text { des personnes qui } \\
\text { utilisent des drogues } \\
\text { par injection de } \\
\text { recevoir des conseils } \\
\text { volontaires fournis par } \\
\text { des pairs et des tests } \\
\text { de dépistage du VIH }\end{array}$ & 4 \\
\hline $\begin{array}{l}\text { O'Byrne \& } \\
\text { Bryan, } 2013 \text { (44) } \\
\text { Ottawa (Ontario) }\end{array}$ & Non indiqué & $\begin{array}{l}\text { Personnes qui } \\
\text { s'identifient } \\
\text { comme gaies, } \\
\text { bisexuelles, } \\
\text { transsexuelles, } \\
\text { bispirituelles, } \\
\text { queers ou en } \\
\text { questionnement }\end{array}$ & 721 & $\begin{array}{l}\text { Moyenne: } \\
37,8 \\
(\mathrm{ET}: 12,1)\end{array}$ & 97,2 & $\begin{array}{l}\text { Quantitative: } \\
\text { Questionnaire } \\
\text { (autoadministré) }\end{array}$ & $\begin{array}{l}\text { Examiner les } \\
\text { pratiques sexuelles } \\
\text { et les antécédents } \\
\text { de dépistage et de } \\
\text { diagnostic des ITS et } \\
\text { du VIH }\end{array}$ & 5 \\
\hline $\begin{array}{l}\text { O'Byrne et al., } \\
2013(1,45) \\
\text { Ottawa (Ontario) }\end{array}$ & Non indiqué & HARSAH & 441 & $\begin{array}{l}\text { Moyenne: } \\
38,0 \\
(E T: 13,1)\end{array}$ & 100 & $\begin{array}{l}\text { Quantitative : } \\
\text { Questionnaire } \\
\text { (autoadministré) }\end{array}$ & $\begin{array}{l}\text { Étudier l'impact des } \\
\text { poursuites pour non- } \\
\text { divulgation et de la } \\
\text { prévention du VIH }\end{array}$ & 5 \\
\hline
\end{tabular}


Tableau 1 : Résumé des études incluses faisant état des obstacles et des facteurs favorables au dépistage du VIH au Canada, 2009 à 2019 (suite)

\begin{tabular}{c|c|c|c|c|c|c|c|c}
$\begin{array}{c}\text { Citation et } \\
\text { emplacement }\end{array}$ & $\begin{array}{c}\text { Années de } \\
\text { collecte } \\
\text { des } \\
\text { données }\end{array}$ & $\begin{array}{c}\text { Population à } \\
\text { l'étude }\end{array}$ & $\begin{array}{c}\text { Taille de } \\
\text { l'échantillon }\end{array}$ & $\begin{array}{c}\text { Âge (en } \\
\text { années) }\end{array}$ & $\begin{array}{c}\text { Hommes } \\
(\%)\end{array}$ & Type d'étude & $\begin{array}{c}\text { Question de } \\
\text { recherche }\end{array}$ & $\begin{array}{c}\text { Note de } \\
\text { qualité } \\
(/ 5)\end{array}$
\end{tabular}

Littérature révisée par les pairs (suite)

\begin{tabular}{|c|c|c|c|c|c|c|c|c|}
\hline $\begin{array}{l}\text { O'Byrne \& } \\
\text { Watts, } 2014 \text { (46) } \\
\text { Ottawa (Ontario) }\end{array}$ & Non indiqué & $\begin{array}{l}\text { Jeunes hommes } \\
\text { homosexuels }\end{array}$ & 8 & $\begin{array}{l}\text { Moyenne : } \\
23,3 \\
\text { (ET : Non } \\
\text { indiqué) }\end{array}$ & 100 & $\begin{array}{l}\text { Qualitative : } \\
\text { Entrevues semi- } \\
\text { structurées avec } \\
\text { analyse thématique }\end{array}$ & $\begin{array}{l}\text { Explorer les } \\
\text { perceptions de la } \\
\text { stigmatisation dans les } \\
\text { soins de santé chez les } \\
\text { jeunes homosexuels }\end{array}$ & 5 \\
\hline $\begin{array}{l}\text { O'Byrne et al., } \\
2013(2,47) \\
\text { Ottawa (Ontario) }\end{array}$ & Non indiqué & HARSAH & 27 & $\begin{array}{l}\text { Catégorie : } \\
19-30 \text { (48 \%), } \\
31-40(30 \%), \\
41-50(13 \%), \\
51-60(9 \%)\end{array}$ & 100 & $\begin{array}{l}\text { Méthodes mixtes: } \\
\text { Entrevues semi- } \\
\text { structurées avec } \\
\text { analyse thématique }\end{array}$ & $\begin{array}{l}\text { Examiner le dépistage } \\
\text { du VIH et les attitudes } \\
\text { des HARSAH à la suite } \\
\text { de communiqués de } \\
\text { presse régionaux au } \\
\text { sujet d'une poursuite } \\
\text { locale pour non- } \\
\text { divulgation }\end{array}$ & 4 \\
\hline $\begin{array}{l}\text { Pai et al., } 2018 \\
(48) \\
\text { Toutes les } \\
\text { provinces }\end{array}$ & 2015 & $\begin{array}{l}\text { Intervenants } \\
\text { participant } \\
\text { aux initiatives } \\
\text { d'autodiagnostic } \\
\text { du VIH au } \\
\text { Canada }\end{array}$ & 183 & Non indiqué & $\begin{array}{l}\text { Non } \\
\text { indiqué }\end{array}$ & $\begin{array}{l}\text { Méthodes mixtes : } \\
\text { Questionnaire } \\
\text { (autoadministré), } \\
\text { questions ouvertes } \\
\text { et commentaires }\end{array}$ & $\begin{array}{l}\text { Cerner les } \\
\text { préoccupations, les } \\
\text { possibilités et les défis } \\
\text { liés à la mise en œuvre } \\
\text { de l'autodépistage du } \\
\text { VIH au Canada }\end{array}$ & 4 \\
\hline $\begin{array}{l}\text { Pai et al., } 2014 \\
\text { (49) } \\
\text { Montréal } \\
\text { (Québec) }\end{array}$ & 2011-2012 & $\begin{array}{l}\text { Étudiants d'une } \\
\text { clinique de santé } \\
\text { universitaire }\end{array}$ & 145 & $\begin{array}{l}\text { Médiane : } 22 \\
\text { (El : Non } \\
\text { indiqué) }\end{array}$ & 39,8 & $\begin{array}{l}\text { Méthodes mixtes: } \\
\text { Questionnaire } \\
\text { (autoadministré), } \\
\text { questions ouvertes }\end{array}$ & $\begin{array}{l}\text { Examiner la faisabilité } \\
\text { d'offrir une stratégie } \\
\text { d'autoévaluation } \\
\text { non supervisée aux } \\
\text { étudiants canadiens }\end{array}$ & 5 \\
\hline $\begin{array}{l}\text { Rich et al., } 2017 \\
\text { (50) } \\
\text { Vancouver } \\
\text { (Colombie- } \\
\text { Britannique) }\end{array}$ & 2012-2014 & $\begin{array}{l}\text { Hommes } \\
\text { transgenres gais, } \\
\text { bisexuels et } \\
\text { queers }\end{array}$ & 11 & $\begin{array}{l}\text { Médiane : } 26 \\
(E I: 25-28)\end{array}$ & 100 & $\begin{array}{l}\text { Qualitative : } \\
\text { Entrevues semi- } \\
\text { structurées avec } \\
\text { analyse thématique }\end{array}$ & $\begin{array}{l}\text { Explorer le } \\
\text { risque sexuel de } \\
\text { transmission du VIH } \\
\text { chez les hommes } \\
\text { transgenres dans } \\
\text { un environnement } \\
\text { d'accès universel } \\
\text { aux soins de santé } \\
\text { financé par le secteur } \\
\text { public, notamment } \\
\text { le dépistage et le } \\
\text { traitement du VIH }\end{array}$ & 5 \\
\hline $\begin{array}{l}\text { Scheim \& } \\
\text { Travers, } 2017 \\
\text { (51) } \\
\text { Ontario }\end{array}$ & 2013 & $\begin{array}{l}\text { HARSAH } \\
\text { transgenres }\end{array}$ & 40 & $\begin{array}{l}\text { Catégorie : } \\
18-24(25 \%), \\
25-34(48 \%), \\
35-44(23 \%), \\
\text { Plus de } 45 \\
(5 \%)\end{array}$ & 100 & $\begin{array}{l}\text { Qualitative : } \\
\text { Entrevues semi- } \\
\text { structurées avec } \\
\text { analyse thématique }\end{array}$ & $\begin{array}{l}\text { Identifier les points de } \\
\text { vue des HARSAH trans } \\
\text { sur les obstacles et les } \\
\text { facteurs favorables au } \\
\text { dépistage du VIH et } \\
\text { des ITS }\end{array}$ & 5 \\
\hline $\begin{array}{l}\text { Stenstrom et al., } \\
2016(52) \\
\text { Vancouver } \\
\text { (Colombie- } \\
\text { Britannique) }\end{array}$ & 2009-2011 & $\begin{array}{l}\text { Patients en } \\
\text { soins tertiaires } \\
\text { d'urgence }\end{array}$ & 1402 & $\begin{array}{l}\text { Moyenne : } \\
43,3 \\
(\text { ET : } 11,6)\end{array}$ & 58,4 & $\begin{array}{l}\text { Quantitative : } \\
\text { Questionnaire } \\
\text { (autoadministré) }\end{array}$ & $\begin{array}{l}\text { Estimer l'acceptabilité } \\
\text { du dépistage du VIH } \\
\text { au point de service } \\
\text { dans un service } \\
\text { d'urgence }\end{array}$ & 4 \\
\hline $\begin{array}{l}\text { Stephenson et } \\
\text { al., } 2014(53) \\
\text { Non spécifié }\end{array}$ & 2011-2012 & $\begin{array}{l}\text { Les utilisateurs } \\
\text { masculins de } \\
\text { Facebook } \\
\text { manifestant un } \\
\text { intérêt pour les } \\
\text { hommes }\end{array}$ & 344 & $\begin{array}{l}\text { Catégorie : } \\
18-24(42 \%), \\
25-34(26 \%), \\
35-44(13 \%), \\
\text { Plus de } 45 \\
(19 \%)\end{array}$ & 100 & $\begin{array}{l}\text { Quantitative : } \\
\text { Questionnaire } \\
\text { (autoadministré) }\end{array}$ & $\begin{array}{l}\text { Examiner les } \\
\text { liens entre les } \\
\text { caractéristiques } \\
\text { individuelles et la } \\
\text { volonté des couples } \\
\text { d'HARSAH d'utiliser } \\
\text { les conseils et le } \\
\text { dépistage volontaires } \\
\text { du VIH chez les } \\
\text { couples }\end{array}$ & 5 \\
\hline
\end{tabular}


Tableau 1 : Résumé des études incluses faisant état des obstacles et des facteurs favorables au dépistage du VIH au Canada, 2009 à 2019 (suite)

\begin{tabular}{|c|c|c|c|c|c|c|c|c|}
\hline $\begin{array}{c}\text { Citation et } \\
\text { emplacement }\end{array}$ & $\begin{array}{l}\text { Années de } \\
\text { collecte } \\
\text { des } \\
\text { données }\end{array}$ & $\begin{array}{c}\text { Population à } \\
\text { l'étude }\end{array}$ & $\begin{array}{c}\text { Taille de } \\
\text { l'échantillon }\end{array}$ & $\begin{array}{l}\text { Âge (en } \\
\text { années) }\end{array}$ & $\begin{array}{l}\text { Hommes } \\
(\%)\end{array}$ & Type d'étude & $\begin{array}{l}\text { Question de } \\
\text { recherche }\end{array}$ & $\begin{array}{c}\text { Note de } \\
\text { qualité } \\
(/ 5)\end{array}$ \\
\hline \multicolumn{9}{|c|}{ Littérature révisée par les pairs } \\
\hline $\begin{array}{l}\text { Worthington et } \\
\text { al., } 2015(54) \\
\text { Toutes les } \\
\text { provinces et } \\
\text { territoires }\end{array}$ & 2011 & $\begin{array}{l}\text { Population } \\
\text { générale }\end{array}$ & 2139 & $\begin{array}{l}\text { Catégorie : } \\
16-29 \\
(23,3 \%), \\
30-59 \\
(50,8 \%), \\
\text { Plus de } 60 \\
(25,9 \%)\end{array}$ & 48,2 & $\begin{array}{l}\text { Quantitative : } \\
\text { Questionnaire } \\
\text { (autoadministré } \\
\text { et administré par } \\
\text { l'intervieweur) }\end{array}$ & $\begin{array}{l}\text { Décrire le dépistage } \\
\text { volontaire du VIH } \\
\text { dans la population } \\
\text { générale et examiner } \\
\text { les connaissances } \\
\text { individuelles, les } \\
\text { comportements } \\
\text { et les facteurs } \\
\text { sociodémographiques } \\
\text { associés au dépistage }\end{array}$ & 5 \\
\hline $\begin{array}{l}\text { Worthington et } \\
\text { al., } 2016(55) \\
\text { Non spécifié }\end{array}$ & Non indiqué & Infirmières & 40 & Non indiqué & $\begin{array}{l}\text { Non } \\
\text { indiqué }\end{array}$ & $\begin{array}{l}\text { Méthodes mixtes: } \\
\text { Entrevues semi- } \\
\text { structurées avec } \\
\text { analyse thématique }\end{array}$ & $\begin{array}{l}\text { Évaluer l'impact } \\
\text { d'une intervention de } \\
\text { mentorat en matière } \\
\text { de soins du VIH sur } \\
\text { les connaissances, } \\
\text { les attitudes et les } \\
\text { pratiques avec les } \\
\text { infirmières et les } \\
\text { personnes vivant avec } \\
\text { le VIH }\end{array}$ & 4 \\
\hline \multicolumn{9}{|l|}{ Littérature grise } \\
\hline $\begin{array}{l}\text { Barbour, } 2017 \\
\text { (56) } \\
\text { Halifax } \\
\text { (Nouvelle- } \\
\text { Écosse) }\end{array}$ & Non indiqué & $\begin{array}{l}\text { Collectivités } \\
\text { autochtones }\end{array}$ & 6 & Non indiqué & 50 & $\begin{array}{l}\text { Qualitative : } \\
\text { Entrevues semi- } \\
\text { structurées avec } \\
\text { analyse thématique }\end{array}$ & $\begin{array}{l}\text { Acquérir des } \\
\text { connaissances } \\
\text { communautaires et } \\
\text { une compréhension } \\
\text { des obstacles et des } \\
\text { facteurs favorables } \\
\text { perçus qui sont } \\
\text { associés à l'accès et } \\
\text { à l'acceptabilité du } \\
\text { dépistage du VIH au } \\
\text { sein des populations } \\
\text { autochtones }\end{array}$ & 5 \\
\hline $\begin{array}{l}\text { CATIE } \\
\text { (Community } \\
\text { AIDS Treatment } \\
\text { Information } \\
\text { Exchange), } 2016 \\
\text { (57) } \\
\text { Toutes les } \\
\text { provinces et } \\
\text { territoires }\end{array}$ & 2016 & $\begin{array}{l}\text { Intervenants } \\
\text { travaillant sur } \\
\text { les programmes } \\
\text { de lutte contre } \\
\text { le VIH }\end{array}$ & 65 & Non indiqué & $\begin{array}{l}\text { Non } \\
\text { indiqué }\end{array}$ & $\begin{array}{l}\text { Qualitative : } \\
\text { Dialogue en } \\
\text { groupe délibératif }\end{array}$ & $\begin{array}{l}\text { Produire des } \\
\text { orientations } \\
\text { prioritaires clés sur le } \\
\text { dépistage du VIH et } \\
\text { les programmes de } \\
\text { liaison pour améliorer } \\
\text { la capacité à atteindre } \\
\text { les personnes qui } \\
\text { ne connaissent } \\
\text { pas encore leur } \\
\text { séropositivité et les } \\
\text { orienter vers les soins }\end{array}$ & 2 \\
\hline $\begin{array}{l}\text { Centre Sida } \\
\text { amitié, } 2019 \text { (58) } \\
\text { Laurentides } \\
\text { (Québec) }\end{array}$ & Non indiqué & $\begin{array}{l}\text { Personnes } \\
\text { vivant avec le } \\
\text { VIH, personnes } \\
\text { qui utilisent } \\
\text { des drogues } \\
\text { par injection, } \\
\text { partenaires } \\
\text { experts }\end{array}$ & 196 & Non indiqué & $\begin{array}{l}\text { Non } \\
\text { indiqué }\end{array}$ & $\begin{array}{l}\text { Qualitative: } \\
\text { Questionnaire } \\
\text { (autoadministré } \\
\text { et administré par } \\
\text { l'intervieweur) }\end{array}$ & $\begin{array}{l}\text { Formuler des } \\
\text { recommandations } \\
\text { pour que les } \\
\text { collectivités atteignent } \\
\text { les cibles } 90-90-90\end{array}$ & 2 \\
\hline $\begin{array}{l}\text { Gahagan et al., } \\
2012(59) \\
\text { Halifax } \\
\text { (Nouvelle- } \\
\text { Écosse) }\end{array}$ & 2011 & $\begin{array}{l}\text { Clients du } \\
\text { Centre de } \\
\text { santé-sexualité } \\
\text { d'Halifax }\end{array}$ & 258 & Non indiqué & $\begin{array}{l}\text { Non } \\
\text { indiqué }\end{array}$ & $\begin{array}{l}\text { Méthodes mixtes: } \\
\text { Questionnaire } \\
\text { (autoadministré), } \\
\text { questions ouvertes }\end{array}$ & $\begin{array}{l}\text { Évaluer le rendement } \\
\text { du Programme de } \\
\text { dépistage anonyme } \\
\text { du VIH, évaluer } \\
\text { l'intérêt des clients } \\
\text { pour le dépistage } \\
\text { rapide du VIH au point } \\
\text { de service et leur } \\
\text { volonté de payer des } \\
\text { frais pour cette option } \\
\text { de dépistage }\end{array}$ & 3 \\
\hline
\end{tabular}


Tableau 1 : Résumé des études incluses faisant état des obstacles et des facteurs favorables au dépistage du VIH au Canada, 2009 à 2019 (suite)

\begin{tabular}{|c|c|c|c|c|c|c|c|c|}
\hline $\begin{array}{c}\text { Citation et } \\
\text { emplacement }\end{array}$ & $\begin{array}{l}\text { Années de } \\
\text { collecte } \\
\text { des } \\
\text { données }\end{array}$ & $\begin{array}{c}\text { Population à } \\
\text { l'étude }\end{array}$ & $\begin{array}{l}\text { Taille de } \\
\text { l'échantillon }\end{array}$ & $\begin{array}{l}\text { Âge (en } \\
\text { années) }\end{array}$ & $\begin{array}{c}\text { Hommes } \\
(\%)\end{array}$ & Type d'étude & $\begin{array}{l}\text { Question de } \\
\text { recherche }\end{array}$ & $\begin{array}{c}\text { Note de } \\
\text { qualité } \\
(/ 5)\end{array}$ \\
\hline \multicolumn{9}{|c|}{ Littérature grise (suite) } \\
\hline $\begin{array}{l}\text { Konkor, } 2019 \\
\text { (60) } \\
\text { London/Ottawa/ } \\
\text { Toronto/Windsor } \\
\text { (Ontario) }\end{array}$ & 2018-2019 & $\begin{array}{l}\text { Hommes } \\
\text { hétérosexuels } \\
\text { des } \\
\text { communautés } \\
\text { africaines, } \\
\text { antillaises et } \\
\text { noires }\end{array}$ & 156 & $\begin{array}{l}\text { Catégorie : } \\
\text { 16-19 (14\%), } \\
20-29(32 \%), \\
30-39(26 \%), \\
40-49(16 \%), \\
\text { Plus de } 50 \\
(12 \%)\end{array}$ & 100 & $\begin{array}{l}\text { Quantitative : } \\
\text { Questionnaire } \\
\text { (autoadministré) }\end{array}$ & $\begin{array}{l}\text { Déterminer les } \\
\text { facteurs qui } \\
\text { influencent le recours } \\
\text { aux services de } \\
\text { dépistage du VIH } \\
\text { chez les hommes } \\
\text { hétérosexuels des } \\
\text { communautés } \\
\text { africaines, antillaises } \\
\text { et noires }\end{array}$ & 4 \\
\hline $\begin{array}{l}\text { Messier-Peet et } \\
\text { al., } 2018 \text { (61) } \\
\text { Montréal } \\
\text { (Québec) }\end{array}$ & 2017-2018 & gbHARSAH & 551 & Non indiqué & 100 & $\begin{array}{l}\text { Quantitative : } \\
\text { Questionnaire } \\
\text { (autoadministré) }\end{array}$ & $\begin{array}{l}\text { Étudier les facteurs } \\
\text { associés à l'absence } \\
\text { de dépistage du VIH } \\
\text { chez les gbHARSAH } \\
\text { à haut risque de } \\
\text { contracter le VIH }\end{array}$ & 4 \\
\hline $\begin{array}{l}\text { Our Youth, Our } \\
\text { Response, } 2014 \\
\text { (62) } \\
\text { Provinces de } \\
\text { l'Atlantique }\end{array}$ & 2011-2013 & $\begin{array}{l}\text { Intervenants du } \\
\text { gouvernement, } \\
\text { des secteurs } \\
\text { communautaires } \\
\text { et de la } \\
\text { recherche, } \\
\text { fournisseurs } \\
\text { de services de } \\
\text { santé et clients } \\
\text { des organismes } \\
\text { communautaires }\end{array}$ & 69 & $\begin{array}{l}\text { Catégorie : } \\
16-25(16 \%), \\
26-35(20 \%), \\
36-45(19 \%), \\
46-55(20 \%), \\
\text { Plus de } 56 \\
(19 \%)\end{array}$ & 45,4 & $\begin{array}{l}\text { Méthodes mixtes: } \\
\text { Entrevues et } \\
\text { groupes de } \\
\text { discussion avec } \\
\text { analyse thématique }\end{array}$ & $\begin{array}{l}\text { Élaborer des } \\
\text { recommandations } \\
\text { fondées sur des } \\
\text { données probantes } \\
\text { à l'intention des } \\
\text { intervenants du } \\
\text { gouvernement, des } \\
\text { collectivités et des } \\
\text { secteurs de recherche } \\
\text { sur les approches } \\
\text { de prévention, } \\
\text { les politiques et } \\
\text { les programmes } \\
\text { nécessaires pour aider } \\
\text { à atténuer l'impact } \\
\text { du VIH et du virus de } \\
\text { l'hépatite C }\end{array}$ & 4 \\
\hline $\begin{array}{l}\text { L'Agence, } 2018 \\
\text { (63) } \\
\text { Toutes les } \\
\text { provinces et } \\
\text { territoires }\end{array}$ & 2010-2012 & $\begin{array}{l}\text { Personnes qui } \\
\text { utilisent des } \\
\text { drogues par } \\
\text { injection }\end{array}$ & 2687 & $\begin{array}{l}\text { Moyenne: } \\
39,4 \\
\text { (ET: Non } \\
\text { indiqué) }\end{array}$ & 68,2 & $\begin{array}{l}\text { Quantitative : } \\
\text { Questionnaire } \\
\text { (administré par } \\
\text { l'intervieweur) }\end{array}$ & $\begin{array}{l}\text { Orienter les efforts } \\
\text { de prévention et } \\
\text { de contrôle du } \\
\text { VIH, l'élaboration } \\
\text { des politiques de } \\
\text { santé publique et } \\
\text { l'évaluation des } \\
\text { programmes }\end{array}$ & 4 \\
\hline $\begin{array}{l}\text { Vannice, } 2016 \\
(64) \\
\text { Ottawa (Ontario) }\end{array}$ & Non indiqué & $\begin{array}{l}\text { Les femmes dans } \\
\text { les communautés } \\
\text { africaines, } \\
\text { antillaises et } \\
\text { noires }\end{array}$ & 10 & $\begin{array}{l}\text { Étendue : } \\
18-60\end{array}$ & 0 & $\begin{array}{l}\text { Qualitative : } \\
\text { Entrevues semi- } \\
\text { structurées avec } \\
\text { analyse thématique }\end{array}$ & $\begin{array}{l}\text { Examiner les } \\
\text { expériences, les } \\
\text { perceptions et } \\
\text { les connaissances } \\
\text { concernant le } \\
\text { dépistage du VIH } \\
\text { chez les femmes } \\
\text { de communautés } \\
\text { africaines, antillaises } \\
\text { et noires }\end{array}$ & 3 \\
\hline $\begin{array}{l}\text { Wertheimer, } \\
2011(6) \\
\text { Toutes les } \\
\text { provinces et } \\
\text { territoires }\end{array}$ & 2009-2010 & $\begin{array}{l}\text { Fournisseurs de } \\
\text { services }\end{array}$ & $\begin{array}{l}\text { Quantitative : } \\
75 \\
\text { Qualitative : } 15\end{array}$ & Non indiqué & $\begin{array}{l}\text { Non } \\
\text { indiqué }\end{array}$ & $\begin{array}{l}\text { Méthodes mixtes : } \\
\text { Questionnaire } \\
\text { (autoadministré en } \\
\text { ligne), entrevues } \\
\text { individuelles }\end{array}$ & $\begin{array}{l}\text { Cerner les obstacles } \\
\text { qui entravent l'accès } \\
\text { des femmes au } \\
\text { dépistage du VIH }\end{array}$ & 2 \\
\hline
\end{tabular}

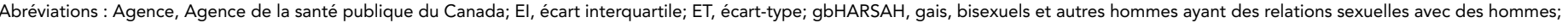

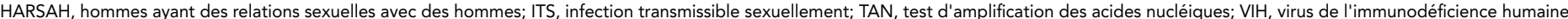

de non-réponse, ce qui est attendu puisque bon nombre de ces études ont été menées auprès de populations difficiles à rejoindre. L'élément le plus faible des études utilisant des méthodes mixtes était le manque de prise en compte de la divergence entre les résultats qualitatifs et quantitatifs. 


\section{Synthèse des résultats}

La synthèse narrative des résultats qui suit résume les obstacles et les facteurs favorables identifiés, de façon globale et en fonction de la population clé et de la province ou du territoire. Les caractéristiques sociodémographiques et les comportements associés au dépistage du VIH sont présentés séparément, car ils représentent des facteurs individuels associés au dépistage plutôt que des obstacles ou facteurs favorables externes.

\section{Aperçu des obstacles et des facteurs favorables au dépistage du VIH}

Au niveau de l'individu, plusieurs obstacles au dépistage du VIH sont ressortis dans plusieurs contextes différents, notamment la peur d'obtenir un résultat positif $(6,25,39,56,58,64)$; la stigmatisation entourant le VIH et les comportements ou identités perçus comme étant associés au VIH $(23,31,38,41,56,58,60,64)$; la perception d'être à faible risque d'exposition au VIH $(6,24,26,50,51,61,62)$; les connaissances insuffisantes du $\mathrm{VIH}$ et des options de dépistage $(56,61,64)$; la difficulté d'accès aux services de dépistage, par exemple, heures d'ouverture limitées de la clinique, difficulté à obtenir un rendez-vous $(23,28,41,58,60,64)$; et le niveau insuffisant de confidentialité des services de dépistage $(28,41,42,56,58,64)$. Certaines caractéristiques sociodémographiques ont été relevées comme étant associées à un dépistage accru, notamment l'adoption de comportements associés au VIH (e.g. augmentation du nombre de partenaires sexuels, consommation de drogues par injection) $(24,27,40,54,60,61,63)$ et le fait d'avoir déjà fait l'objet d'un test de dépistage des ITSS $(24,25,38)$.

Au niveau des fournisseurs de soins de santé, les obstacles communs relevés comprenaient la stigmatisation liée au $\mathrm{VIH}$ chez les fournisseurs de soins de santé $(46,57)$, la perception qu'un patient est à faible risque d'exposition au VIH $(6,64)$ et la réticence ou le refus d'offrir le dépistage à des personnes qui n'étaient pas perçues comme étant à risque $(38,58)$. Selon de nombreuses études, la pratique d'offrir un test de dépistage du $\mathrm{VIH}(25,26,58)$ et des pratiques non stigmatisantes en matière de soins de santé $(23,50,51)$ étaient des facteurs favorables au dépistage.

Au niveau institutionnel ou politique, la criminalisation de certains comportements (e.g. travail du sexe, consommation de drogues, non-divulgation du VIH) $(23,57)$ et le manque de ressources et d'infrastructures de santé adéquates dans les régions rurales et éloignées $(28,56,58,62)$ représentent des obstacles structurels au dépistage. À l'inverse, les politiques et les pratiques institutionnelles qui augmentent l'accessibilité, la commodité et la confidentialité des tests (e.g. vaste éventail d'options de test, réduction des temps d'attente, test à faible coût) $(6,23,25-27,29,41,49-51,58,62)$ et intègrent les tests aux services de soins de santé courants $(25,31,38,51,58,63,64)$, les campagnes éducatives et promotionnelles $(6,28,32,62,64)$ et la collaboration intersectorielle $(6,28,62)$ ont été déclarées comme des facteurs favorables au dépistage.

\section{Résultats par population clé}

Beaucoup d'études portaient sur la population gbHARSAH ( $n=15$ ) $(24,32-34,36-38,44-47,50,51,53,61)$, reflétant l'épidémiologie historique du VIH au Canada. Les autres principales populations comprennent les professionnels du sexe $(n=2)(23,27)$, les personnes qui utilisent des drogues par injection $(n=3)(43,58,63)$, les populations d'immigrants $(n=3)(23,41,60)$, les collectivités autochtones $(n=1)(56)$ et les communautés africaines, antillaises et noires $(n=2)(60,64)$. Le tableau 2 résume les résultats par population clé pour mettre en évidence les besoins particuliers de chaque population et leur contexte.

\section{Tableau 2 : Obstacles et facteurs favorables au dépistage du VIH par population clé au Canada, 2009 à 2019}

\begin{tabular}{|c|c|c|c|}
\hline $\begin{array}{c}\text { Type de } \\
\text { population }\end{array}$ & $\begin{array}{l}\text { Provinces } \\
\text { traitant de la } \\
\text { population }\end{array}$ & Obstacles & Facteurs favorables \\
\hline $\begin{array}{l}\text { gbHARSAH } \\
\text { (y compris les } \\
\text { personnes } \\
\text { bispirituelles, } \\
\text { queers, } \\
\text { trans ou en } \\
\text { questionnement) }\end{array}$ & $\begin{array}{l}\text { Toutes les } \\
\text { provinces }\end{array}$ & $\begin{array}{l}\text { - Crainte d'un résultat positif (51) } \\
\text { - Honte associée au fait de demander un test de } \\
\text { dépistage du VIH et de répondre au questionnaire } \\
\text { préalable au test (e.g. divulgation d'information } \\
\text { sexuelle) (41) } \\
\text { - Manque de tests anonymes }(44,47) \\
\text { - Manque de confidentialité dans les services de } \\
\text { dépistage (41) } \\
\text { - Manque de connaissances sur les identités trans et les } \\
\text { préoccupations liées à la santé chez les fournisseurs de } \\
\text { tests (51) } \\
\text { - Disponibilité et accessibilité limitées du dépistage } \\
\text { du VIH (31) (e.g. heures d'ouverture limitées des } \\
\text { cliniques (41)) } \\
\text { - Perception d'un faible risque d'acquisition ou de } \\
\text { transmission du VIH (24,50,51) } \\
\text { - Criminalisation de la non-divulgation du VIH }(36,45,47) \\
\text { - Stigmatisation et discrimination liées au genre, à la } \\
\text { sexualité, à l'identité sexuelle, aux relations sexuelles et } \\
\text { à la monogamie }(31) \\
\text { - Stigmatisation par les professionnels de la santé }(46)\end{array}$ & $\begin{array}{l}\text { - Avoir un solide réseau parmi les gbHARSAH dans la } \\
\text { collectivité (50) } \\
\text { - Soins de santé sexuelle adaptés aux gbHARSAH, aux } \\
\text { - Intégration queers et aux personnes trans (50) } \\
\text { santé de base (31) } \\
\text { - } \quad \text { Dépistage du VIH à d'autres services de } \\
\text { - Campagnes dans les lnternet (33) } \\
\text { dépistage du VIH (32) }\end{array}$ \\
\hline
\end{tabular}


Tableau 2 : Obstacles et facteurs favorables au dépistage du VIH par population clé au Canada, 2009 à 2019 (suite)

\begin{tabular}{|c|c|c|c|}
\hline $\begin{array}{l}\text { Type de } \\
\text { population }\end{array}$ & $\begin{array}{l}\text { Provinces } \\
\text { traitant de la } \\
\text { population }\end{array}$ & Obstacles & Facteurs favorables \\
\hline $\begin{array}{l}\text { Travailleurs } \\
\text { du sexe (y } \\
\text { compris les } \\
\text { gestionnaires et } \\
\text { les propriétaires } \\
\text { d'entreprises de } \\
\text { commerce du } \\
\text { sexe) }\end{array}$ & $\begin{array}{l}\text { Colombie- } \\
\text { Britannique }\end{array}$ & $\begin{array}{l}\text { - Criminalisation du travail du sexe (23) } \\
\text { - Criminalisation de tierces parties (gestionnaires/ } \\
\text { propriétaires) créant des pratiques préjudiciables } \\
\text { dans les lieux de travail du sexe (e.g. restrictions sur } \\
\text { l'utilisation du condom, rejet des tests en milieu de } \\
\text { travail) (23) } \\
\text { - La collaboration entre les intervenants en santé publique } \\
\text { et les organismes d'application de la loi (e.g. arrivée sur } \\
\text { place ensemble) a entraîné une méfiance à l'égard des } \\
\text { intervenants en santé et une réticence à les laisser venir } \\
\text { sur place (23) } \\
\text { - Stigmatisation professionnelle entraînant des difficultés } \\
\text { d'accès aux soins de santé primaires et aux services de } \\
\text { santé sexuelle (23) } \\
\text { - Crainte que leur emploi de travailleur du sexe soit connu } \\
\text { (e.g. réticence à demander des examens fréquents aux } \\
\text { médecins de famille) (23) }\end{array}$ & $\begin{array}{l}\text { - Programmes mobiles de prévention du VIH (27) } \\
\text { - Travailleurs de la santé offrant des tests de dépistage des } \\
\text { ITSS sur les lieux de travail du sexe (23) } \\
\text { - Attitudes sans jugement et non stigmatisantes chez les } \\
\text { travailleurs de sensibilisation dans le domaine de la santé } \\
\text { permettant des discussions ouvertes sur les questions de } \\
\text { santé sexuelle (23) }\end{array}$ \\
\hline $\begin{array}{l}\text { Personnes qui } \\
\text { utilisent des } \\
\text { drogues par } \\
\text { injection }\end{array}$ & $\begin{array}{l}\text { Toutes les } \\
\text { provinces }\end{array}$ & $\begin{array}{l}\text { - Faible perception des risques, manque d'intérêt ou de } \\
\text { sentiment d'urgence (63) } \\
\text { - Crainte d'un diagnostic positif (63) } \\
\text { - Se sentir en bonne santé (63) } \\
\text { - Problèmes liés à la réalisation de tests de dépistage (e.g. } \\
\text { - S'accessibilité des services de test) (63) } \\
\text { Pentiment que rien ne peut être fait en cas de diagnostic } \\
\text { positif (63) }\end{array}$ & $\begin{array}{l}\text { - Conseils dispensés par des pairs après avoir passé un } \\
\text { - test (43) } \\
\text { - Ve soumettre régulièrement à un test de dépistage du } \\
\text { - Dépistage intégré aux soins médicaux de base (63) } \\
\text { - Dépistage suggéré par le fournisseur de soins de } \\
\text { - santé (63) } \\
\text { - Exposition potentielle récente (e.g. rapports sexuels, } \\
\text { consommation de drogues) (63) }\end{array}$ \\
\hline $\begin{array}{l}\text { Populations } \\
\text { d'immigrants }\end{array}$ & $\begin{array}{l}\text { Colombie- } \\
\text { Britannique, } \\
\text { Ontario, Québec }\end{array}$ & $\begin{array}{l}\text { - Honte associée au fait de demander un test de } \\
\text { dépistage du VIH et de répondre au questionnaire } \\
\text { préalable au test (e.g. divulgation d'information } \\
\text { sexuelle) (41) } \\
\text { - Préoccupations au sujet de la confidentialité (e.g. être } \\
\text { vu à la clinique ou recevoir des services d'un membre } \\
\text { de leur communauté qui est très unie, préférence de } \\
\text { répondre aux questions sur papier ou sur des appareils } \\
\text { électroniques) }(41) \\
\text { - Difficultés d'accès aux soins de santé primaire et aux } \\
\text { services de santé sexuelle en raison de l'absence } \\
\text { d'assurance-maladie, des obstacles linguistiques et } \\
\text { culturels }(23,27,41,60)\end{array}$ & $\begin{array}{l}\text { - Disponibilité de traducteurs ou de services de santé } \\
\text { multilingues (23) }\end{array}$ \\
\hline $\begin{array}{l}\text { Collectivités } \\
\text { autochtones }\end{array}$ & Nouvelle-Écosse & $\begin{array}{l}\text { - Obstacles géographiques à l'accès aux soins de santé } \\
\text { dans les collectivités rurales et éloignées; absence de } \\
\text { soins de santé primaires et de services de dépistage du } \\
\text { VIH; accès irrégulier au transport médical (56) } \\
\text { - Manque de confiance entre les clients et les fournisseurs } \\
\text { de soins de santé (56) } \\
\text { - Manque de connaissances sur le VIH (facteurs de } \\
\text { risque, stratégies de réduction des risques, modes } \\
\text { de transmission, traitement) et le dépistage du VIH } \\
\text { (faisabilité, types disponibles, avantages) (56) } \\
\text { - Stigmatisation du VIH liée à l'utilisation des drogues par } \\
\text { injection (56) } \\
\text { - Perception de faible risque; déni du risque lié à certains } \\
\text { comportements (e.g. consommation de drogues } \\
\text { injectables) (56) } \\
\text { - Peur des résultats positifs et de la perte de l'acceptation } \\
\text { de la collectivité (56) } \\
\text { - Stigmatisation et homophobie; perception du VIH } \\
\text { comme " maladie gaie ", associations avec promiscuité } \\
\text { sexuelle, hiérarchie des comportements stigmatisés, plus } \\
\text { de stigmatisation sociale est associée à l'homosexualité } \\
\text { qu'à l'utilisation des drogues par injection, liée à une } \\
\text { perception différentielle du virus de l'hépatite C et du } \\
\text { VIH (56) } \\
\text { - Problèmes de confidentialité dans les petites } \\
\text { collectivités, croyance que « les gens le sauront » (56) }\end{array}$ & $\begin{array}{l}\text { - La normalisation du dépistage du VIH accroît à la fois } \\
\text { l'accessibilité et l'acceptabilité; on délaisse le dépistage } \\
\text { ciblé fondé sur le comportement, la sexualité et le risque } \\
\text { au profit de l'intégration du dépistage dans les soins } \\
\text { médicaux de base (56) } \\
\text { - Accroître la disponibilité du dépistage; offrir le dépistage } \\
\text { du VIH dans les réserves autochtones; accroître l'accès } \\
\text { au transport médical (56) } \\
\text { - Réduire le temps d'attente pour les résultats en offrant } \\
\text { des tests au point d'intervention (56) } \\
\text { - Centres de services de réduction des méfaits intégrant le } \\
\text { dépistage du VIH (56) } \\
\text { - Éducation sur le VIH (modes de transmission, facteurs } \\
\text { de risque) et le dépistage du VIH (types disponibles, } \\
\text { dépistage en tant que prévention); séances offertes par } \\
\text { des organisations de services de lutte contre le VIH/ } \\
\text { sida (56) } \\
\text { - Collaboration entre les fournisseurs de soins de santé } \\
\text { et les organismes de services de lutte contre le VIH/sida } \\
\text { pour renforcer la confiance (56) } \\
\text { - Pratiques et protocoles acceptables pour la } \\
\text { collectivité (56) } \\
\text { - Éducation combinée sur les autres ITSS (e.g. virus de } \\
\text { I'hépatite C) (56) }\end{array}$ \\
\hline
\end{tabular}


Tableau 2 : Obstacles et facteurs favorables au dépistage du VIH par population clé au Canada, 2009 à 2019 (suite)

\begin{tabular}{|c|c|c|c|}
\hline $\begin{array}{c}\text { Type de } \\
\text { population }\end{array}$ & $\begin{array}{l}\text { Provinces } \\
\text { traitant de la } \\
\text { population }\end{array}$ & Obstacles & Facteurs favorables \\
\hline $\begin{array}{l}\text { Communautés } \\
\text { africaines, } \\
\text { antillaises et } \\
\text { noires }\end{array}$ & Ontario & $\begin{array}{l}\text { - Obstacles culturels (étiquetage des femmes qui } \\
\text { passent des tests en tant que personnes portées à la } \\
\text { promiscuité sexuelle) (64) } \\
\text { - Difficulté d'accès aux installations de santé et de } \\
\text { dépistage (ignorer où obtenir un test de dépistage du } \\
\text { VIH) }(60,64) \\
\text { - Peur des résultats positifs; préférer ne pas savoir (64) } \\
\text { - Crainte d'une réaction négative de la part } \\
\text { des partenaires lors de la divulgation de l'état } \\
\text { sérologique (64) } \\
\text { - Manque de tests anonymes (64) } \\
\text { - Manque de confidentialité dans les services de } \\
\text { - Cópistage du VIH (64) } \\
\text { - Cónnaissance insuffisante du VIH (transmission, } \\
\text { - Stigmatisation et discrimination à l'égard des } \\
\text { comportements sexuels entre personnes de même sexe, } \\
\text { des personnes qui utilisent des drogues par injection ou } \\
\text { des consommateurs d'alcool, idée fausse selon laquelle } \\
\text { le dépistage suppose une faible masculinité }(60,64) \\
\text { - Résistance du médecin de famille à offrir un test de } \\
\text { dépistage malgré la demande }(60,64) \\
\text { - Perception d'offre de test comme une forme de } \\
\text { stéréotype ou de profilage }(60,64)\end{array}$ & $\begin{array}{l}\text { - Examen offert par un médecin de famille dans le } \\
\text { contexte des soins courants (plutôt que d'avoir à le } \\
\text { demander expressément) (64) } \\
\text { - Éliminer la stigmatisation en normalisant le dépistage du } \\
\text { VIH (64) } \\
\text { - Stratégies axées sur l'ouverture de la communication qui } \\
\text { abordent les silences culturels (veiller à ce que les gens } \\
\text { aient les moyens d'aborder le sujet du dépistage du } \\
\text { VIH) (64) } \\
\text { - Témoignages de personnes atteintes du sida ou du VIH } \\
\text { dans le but de réduire la peur liée au dépistage (64) } \\
\text { - Sensibilisation communautaire par des personnes } \\
\text { d'origines culturelles ou linguistiques semblables (64) } \\
\text { - Accroître les connaissances sur le traitement et les } \\
\text { résultats, les recommandations relatives aux tests et les } \\
\text { stratégies de réduction des risques (64) } \\
\text { - Messages de santé publique du gouvernement et des } \\
\text { organismes de santé, en tirant parti des médias grand } \\
\text { public (64) }\end{array}$ \\
\hline
\end{tabular}

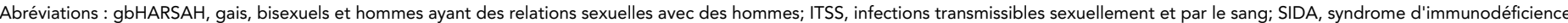
acquise: $\mathrm{VIH}$, virus de l'immunodéficience humaine

Parmi les populations clés, plusieurs obstacles au dépistage du $\mathrm{VIH}$ communs se sont révélés, dont la crainte d'un diagnostic positif $(23,41,51,56,64)$, des expériences de stigmatisation liée au $\mathrm{VIH}(41,56)$, de la perception d'être à faible risque d'exposition au $\mathrm{VIH}(24,50,51,56,63)$, de l'accessibilité limitée aux services de dépistage $(23,27,41,56,60,64)$ et du manque de connaissances sur le VIH $(56,64)$. D'autres obstacles courants représentent des entraves particulièrement importantes au dépistage pour les populations marginalisées, notamment la stigmatisation liée aux comportements ou aux identités perçus comme étant associés au VIH (e.g. comportements sexuels, orientation sexuelle, travail du sexe, consommation de drogues par injection) $(23,24,31,41,46,50,51,56,60,64)$ et la confidentialité insuffisante des services de dépistage. Cela comprend l'absence de tests anonymes et les préoccupations au sujet de la protection de la vie privée dans les petites collectivités ou les collectivités éloignées $(23,41,44,47,56,64)$.

Certains obstacles étaient propres aux populations clés. Les lois qui criminalisent la non-divulgation du VIH et le travail du sexe sont des obstacles au dépistage parmi la communauté gbHARSAH $(36,45,47)$ et les professionnels du sexe $(23)$, respectivement. De plus, les fournisseurs de soins de santé ne connaissent pas suffisamment les préoccupations et les besoins en matière de santé de certaines populations (e.g. les gbHARSAH, les transgenres, les professionnels du sexe), ce qui constitue un obstacle au dépistage dans ces populations $(23,51,56,60)$. De nombreuses populations sont également confrontées à des problèmes distincts d'accessibilité, comme la disponibilité limitée de services de santé multilingues et le manque d'accès à l'assurance-maladie parmi les populations immigrantes $(23,41,60)$, en plus d'obstacles géographiques aux soins de santé dans les collectivités autochtones rurales et éloignées (56).

Malgré la diversité des contextes de ces populations, plusieurs facteurs communs qui sont favorables au dépistage sont apparus, notamment le fait d'offrir le dépistage du VIH avec un large éventail de modalités (e.g. dépistage anonyme, auto dépistage non supervisé) et de contextes (e.g. cliniques mobiles, dépistage au point d'intervention) $(23,27,33,56)$ ainsi que l'intégration des membres des populations clés ayant une expérience vécue (e.g. services de consultation après les tests fournis par les pairs, initiatives de sensibilisation communautaire) $(43,56,64)$. Ce sont des facteurs qui ont souvent été mentionnés comme des moyens d'améliorer l'accessibilité et l'acceptabilité des services de dépistage du VIH pour les populations clés.

Enfin, certains facteurs favorables étaient particulièrement pertinents pour certaines populations clés. Les pratiques de soins de santé inclusives et non stigmatisantes ont été mentionnées par les communautés queer et transgenre comme des facteurs importants qui facilitent le dépistage $(50,51)$. La disponibilité de traducteurs ou de services de santé multilingues a facilité l'accès au dépistage pour les populations immigrantes (23). Au sein des communautés africaines, antillaises et noires, le fait de permettre de liens sociaux avec des personnes vivant avec le VIH et les initiatives éducatives s'attaquant au silence culturel autour du $\mathrm{VIH}$ étaient des facteurs favorables au dépistage (64). 


\section{Résultats par province ou territoire}

Les caractéristiques sociodémographiques relevées qui sont associées au dépistage du $\mathrm{VIH}$, ainsi que les obstacles et les facteurs favorables au dépistage du VIH sont résumés par province ou territoire dans le tableau 3.

Tableau 3 : Caractéristiques sociodémographiques associées à l'augmentation du dépistage du VIH, aux obstacles et aux facteurs favorables au dépistage du VIH, par province ou territoire au Canada, 2009 à 2019

\begin{tabular}{|c|c|c|c|}
\hline Province/territoire & Niveau de l'individu & $\begin{array}{l}\text { Niveau du fournisseur de } \\
\text { soins de santé }\end{array}$ & Niveau des politiques \\
\hline \multicolumn{4}{|l|}{ Colombie-Britannique } \\
\hline $\begin{array}{l}\text { Caractéristiques et comportements } \\
\text { sociodémographiques associés à un } \\
\text { dépistage accru du VIH }\end{array}$ & $\begin{array}{l}\text { - Plus jeune }(24,34) \\
\text { - Etre plus instruit (34) } \\
\text { - } \text { Race/origine ethnique blanche (24) } \\
\text { - } \text { Ayant en milieu urbain }(24,50) \\
\text { à risque (augmentation du } \\
\text { nombre de partenaires et de } \\
\text { pratiques sexuelles anales, } \\
\text { utilisation incohérente du } \\
\text { préservatif, ne pas pratiquer } \\
\text { la sérodiscrimination }(24,27), \\
\text { personnes qui utilisent des drogues } \\
\text { par injection (27)) }\end{array}$ & - s. o. & - s.o. \\
\hline Obstacles & $\begin{array}{l}\text { - Stigmatisation du travail du } \\
\text { - Sexe (23) } \\
\text { - Statut d'immigrant (manque } \\
\text { d'accès à l'assurance-maladie, } \\
\text { obstacles linguistiques et culturels) } \\
\text { (23,24,27) } \\
\text { - Perception d'un faible risque } \\
\text { (d'acquisition ou de transmission du } \\
\text { VIH) }(24,26,50) \\
\text { - Homophobie intériorisée (34) }\end{array}$ & - s.o. & $\begin{array}{l}\text { - Criminalisation du travail du } \\
\text { sexe (23) } \\
\text { - La collaboration entre les } \\
\text { organismes de santé publique et } \\
\text { les organismes d'application de la } \\
\text { loi crée de la méfiance à l'égard } \\
\text { des travailleurs de proximité des } \\
\text { services de santé (23) }\end{array}$ \\
\hline Facteurs favorables & $\begin{array}{l}\text { - Avoir un solide réseau dans la } \\
\text { communauté gbHARSAH (50) } \\
\text { - Avoir déjà été testé pour d'autres } \\
\text { ITSS (24) }\end{array}$ & $\begin{array}{l}\text { - Connaissances gbHARSAH, } \\
\text { queer et trans en matière de } \\
\text { santé sexuelle et de dépistage du } \\
\text { VIH (50) } \\
\text { - Dépistage du VIH lancé/offert } \\
\text { par les fournisseurs de soins de } \\
\text { santé (26) } \\
\text { - Attitude non critique et non } \\
\text { stigmatisante des fournisseurs de } \\
\text { soins de santé (23) }\end{array}$ & $\begin{array}{l}\text { - Disponibilité de traducteurs ou de } \\
\text { services de santé multilingues (23) } \\
\text { - Programmes mobiles de prévention } \\
\text { du VIH (27) } \\
\text { - Test pratique et peu coûteux (e.g. } \\
\text { gratuit, réception des résultats sur } \\
\text { place }(26,30)) \\
\text { - Proposer diverses modalités de } \\
\text { dépistage du VIH : prélèvement } \\
\text { buccal (26), conseil et dépistage } \\
\text { volontaire du VIH pour les } \\
\text { couples (53), conseil après les tests } \\
\text { offerts par les pairs (43) } \\
\text { - Offrir le dépistage du VIH dans } \\
\text { différents contextes: lieux de } \\
\text { travail du sexe (23), cliniques } \\
\text { d'hygiène dentaire (26,30), services } \\
\text { d'urgence (52) } \\
\text { Campagnes dans les médias } \\
\text { sociaux pour promouvoir le } \\
\text { dépistage du VIH (32) }\end{array}$ \\
\hline \multicolumn{4}{|l|}{ Manitoba } \\
\hline Obstacles & $\begin{array}{l}\text { - Peur des résultats positifs; préfère } \\
\text { ne pas savoir (39) } \\
\text { - Perception d'un faible risque (39) }\end{array}$ & - s.o. & - s.o. \\
\hline
\end{tabular}


Tableau 3 : Caractéristiques sociodémographiques associées à l'augmentation du dépistage du VIH, aux obstacles et aux facteurs favorables au dépistage du VIH, par province ou territoire au Canada, 2009 à 2019 (suite)

\section{Province/territoire \\ Niveau de l'individu \\ Niveau du fournisseur de soins de santé \\ Niveau des politiques}

\section{Ontario}

\begin{tabular}{|c|c|c|c|}
\hline $\begin{array}{l}\text { Caractéristiques et comportements } \\
\text { sociodémographiques associés à un } \\
\text { dépistage accru du VIH }\end{array}$ & $\begin{array}{l}\text { - Âge avancé (40) } \\
\text { - Sexe/genre masculin (40) } \\
\text { - Avoir plus d'expérience en matière } \\
\text { - Être immigrant (60) } \\
\text { - Emploi à temps plein; revenu plus } \\
\text { - élevé }(60) \\
\text { - Adopter des comportements à } \\
\text { risque (utilisation de préservatifs, } \\
\text { avoir plusieurs partenaires sexuels, } \\
\text { consommation de drogues par } \\
\text { injection, travail du sexe, avoir } \\
\text { passé du temps en prison, } \\
\text { consommation de drogues en } \\
\text { prison) }(40,60)\end{array}$ & - s.o. & - s.o. \\
\hline Obstacles & 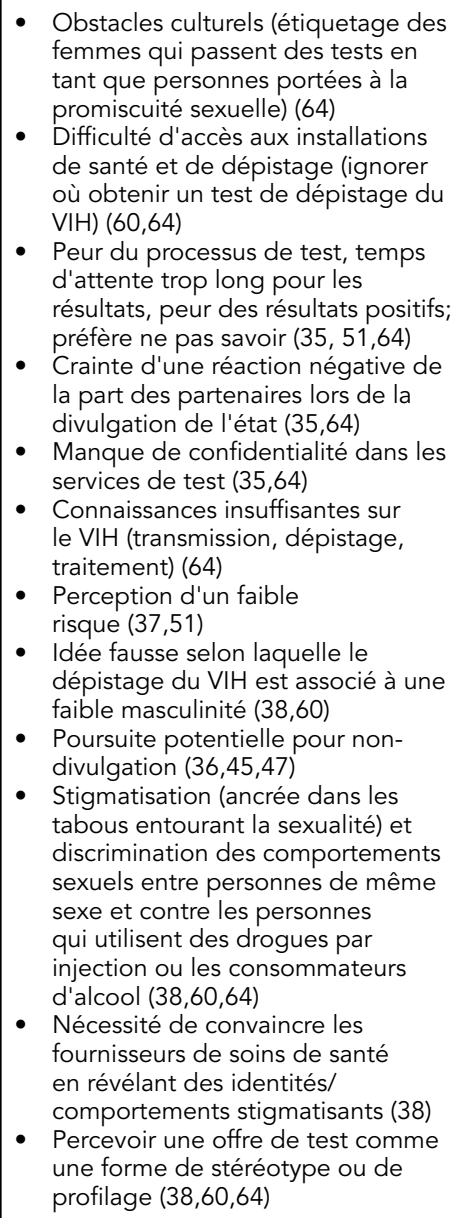 & $\begin{array}{l}\text { - Manque de connaissances sur les } \\
\text { identités trans et les préoccupations } \\
\text { liées à la santé chez les fournisseurs } \\
\text { de soins de santé (51) } \\
\text { - Stigmatisation des professionnels } \\
\text { de la santé (46) } \\
\text { - Perception de faible risque chez les } \\
\text { fournisseurs de soins de santé (64) }\end{array}$ & - s.o. \\
\hline Facteurs favorables & $\begin{array}{l}\text { - } \text { Tests anonymes }(44,47,64) \\
\text { - } \text { Plus d'informations sur le processus } \\
\text { de test }(35) \\
\text { - Plus d'informations sur la } \\
\text { transmission du VIH de la mère à } \\
\text { l'enfant }(35) \\
\text { - } \text { Approche de prévention } \\
\text { personnalisée (35) }\end{array}$ & $\begin{array}{l}\text { - Accès à des testeurs de } \\
\text { confiance (51) } \\
\text { - Interventions sexospécifiques (51) }\end{array}$ & $\begin{array}{l}\text { - Intégration du dépistage du } \\
\text { VIH dans les soins de routine } \\
\text { (déstigmatiser et normaliser le } \\
\text { dépistage du VIH) }(38,51,64) \\
\text { - Accroître les connaissances et } \\
\text { l'éducation sur le VIH dans la } \\
\text { communauté (e.g. par la télévision } \\
\text { et la radio), en particulier auprès } \\
\text { des agences de santé publique (64) } \\
\text { - Établir des liens sociaux avec les } \\
\text { personnes vivant avec le VIH (64) }\end{array}$ \\
\hline
\end{tabular}


Tableau 3 : Caractéristiques sociodémographiques associées à l'augmentation du dépistage du VIH, aux obstacles et aux facteurs favorables au dépistage du VIH, par province ou territoire au Canada, 2009 à 2019 (suite)

\begin{tabular}{|c|c|c|c|}
\hline Province/territoire & Niveau de l'individu & $\begin{array}{l}\text { Niveau du fournisseur de } \\
\text { soins de santé }\end{array}$ & Niveau des politiques \\
\hline \multicolumn{4}{|l|}{ Québec } \\
\hline $\begin{array}{l}\text { Caractéristiques et comportements } \\
\text { sociodémographiques associés à un } \\
\text { dépistage accru du VIH }\end{array}$ & $\begin{array}{l}\text { - Plus grand nombre de partenaires } \\
\text { sexuels (61) }\end{array}$ & - s. o. & - s. o. \\
\hline Obstacles & $\begin{array}{l}\text { - Crainte d'obtenir un résultat } \\
\text { positif, d'être jugé ou rejeté, } \\
\text { et de divulguer le statut aux } \\
\text { partenaires (58) } \\
\text { - Honte associée au fait de } \\
\text { demander un test de dépistage } \\
\text { du VIH et de répondre au } \\
\text { questionnaire préalable au test } \\
\text { (e.g. divulgation d'information } \\
\text { sexuelle) (41,58) } \\
\text { - Manque de confidentialité dans les } \\
\text { - } \text { services de test (41,58) } \\
\text { dennaissance insuffisante } \\
\text { recommices, des lieux et des } \\
\text { dépistage du VIH en (61) } \\
\text { - Accès limité aux fournisseurs de } \\
\text { soins de santé (61) } \\
\text { - Heures d'ouverture limitées des } \\
\text { cliniques de dépistage du VIH (41) } \\
\text { - Perception d'un faible risque (61) } \\
\text { - Tests non couverts par l'assurance- } \\
\text { maladie publique (58) } \\
\text { - Stigmatisation liée au VIH (58) } \\
\end{array}$ & - s. o. & $\begin{array}{l}\text { - Manque de ressources en santé } \\
\text { dans les régions rurales (58) }\end{array}$ \\
\hline Facteurs favorables & - s. o. & $\begin{array}{l}\text { - Les fournisseurs de soins de santé } \\
\text { ne refusent jamais une demande de } \\
\text { dépistage du VIH d'un patient (58) } \\
\text { - Test oral autoadministré non } \\
\text { supervisé (48) }\end{array}$ & $\begin{array}{l}\text { - Intégration du dépistage du VIH } \\
\text { aux soins de santé de routine sans } \\
\text { questionnaire préalable au test (e.g } \\
\text { sur les comportements sexuels) (58) } \\
\text { - Des services de dépistage } \\
\text { accessibles, confidentiels et } \\
\text { pratiques (sans rendez-vous), y } \\
\text { compris des tests non nominatifs et } \\
\text { des tests rapides }(29,41,58) \\
\text { - Offrir diverses modalités de } \\
\text { dépistage du VIH : autodépistage } \\
\text { oral non supervisé (49) } \\
\text { - Offrir des tests de dépistage du } \\
\text { VIH dans divers contextes : dans la } \\
\text { communauté, à la pharmacie (58) } \\
\text { - Efforts de prévention fondés sur les } \\
\text { principes de réduction des méfaits, } \\
\text { axés sur la personne et le virus (58) } \\
\text { - Contexte sécuritaire de dépistage } \\
\text { du VIH (58) }\end{array}$ \\
\hline \multicolumn{4}{|l|}{ Nouvelle-Écosse } \\
\hline $\begin{array}{l}\text { Caractéristiques et comportements } \\
\text { sociodémographiques associés à un } \\
\text { dépistage accru du VIH }\end{array}$ & - Sexe/genre féminin (31) & - s. o. & - s. o. \\
\hline Obstacles & $\begin{array}{l}\text { - Crainte d'un résultat positif au } \\
\text { test, de rejet et d'être associé } \\
\text { à la promiscuité sexuelle et à } \\
\text { l'utilisation des drogues par } \\
\text { injection }(56) \\
\text { - Manque de confidentialité dans les } \\
\text { services de test }(42,56) \\
\text { - Connaissances insuffisantes sur le } \\
\text { VIH et le dépistage }(56) \\
\text { - Stigmatisation et discrimination } \\
\text { liées au genre, à la sexualité, à } \\
\text { l'identité sexuelle, aux relations } \\
\text { sexuelles et à la monogamie }(31,56)\end{array}$ & - s.o. & $\begin{array}{l}\text { - Obstacles géographiques } \\
\text { à l'accès aux soins de santé } \\
\text { dans les collectivités rurales et } \\
\text { éloignées; absence de soins de } \\
\text { santé primaires et de services de } \\
\text { dépistage du VIH dans les petites } \\
\text { collectivités; accès irrégulier au } \\
\text { transport médical (56) }\end{array}$ \\
\hline
\end{tabular}


Tableau 3 : Caractéristiques sociodémographiques associées à l'augmentation du dépistage du VIH, aux obstacles et aux facteurs favorables au dépistage du VIH, par province ou territoire au Canada, 2009 à 2019 (suite)

\begin{tabular}{|c|c|c|c|}
\hline Province/territoire & Niveau de l'individu & $\begin{array}{l}\text { Niveau du fournisseur de } \\
\text { soins de santé }\end{array}$ & Niveau des politiques \\
\hline \multicolumn{4}{|l|}{ Nouvelle-Écosse (suite) } \\
\hline Facteurs favorables & $\begin{array}{l}\text { - Accroître la disponibilité et } \\
\text { l'accessibilité des services de } \\
\text { dépistage du VIH }(31,56) \\
\text { - Être en mesure de payer pour des } \\
\text { tests au point de service (42) }\end{array}$ & - s.o. & $\begin{array}{l}\text { - Intégration du dépistage du VIH } \\
\text { aux services de santé courants (e.g. } \\
\text { dépistage prénatal systématique du } \\
\text { VIH) (31) } \\
\text { - Normalisation du dépistage du } \\
\text { VIH (56) } \\
\text { - Disponibilité de tests rapides (42) }\end{array}$ \\
\hline
\end{tabular}

Terre-Neuve-et-Labrador

\begin{tabular}{|c|c|c|c|}
\hline $\begin{array}{l}\text { Caractéristiques et comportements } \\
\text { sociodémographiques associés à un } \\
\text { dépistage accru du VIH }\end{array}$ & $\begin{array}{l}\text { - HARSAH (les hommes } \\
\text { hétérosexuels reçoivent leur } \\
\text { diagnostic plus tard que les } \\
\text { HARSAH) (25) }\end{array}$ & - s.o. & - s.o. \\
\hline Obstacles & $\begin{array}{l}\text { - Contexte hospitalier (e.g. les } \\
\text { patients dans les cliniques d'ITSS } \\
\text { qui reçoivent leur diagnostic plus } \\
\text { tôt que ceux dans les hôpitaux) (25) } \\
\text { - Peur du diagnostic; déni du } \\
\text { risque (25) } \\
\text { - Interactions négatives avec le } \\
\text { système de santé (25) } \\
\text { - Stigmatisation entourant le VIH et } \\
\text { le dépistage }\end{array}$ & - s.o. & $\begin{array}{l}\text { - Connaissance insuffisante du VIH } \\
\text { dans la population générale (peur } \\
\text { du VIH, idées fausses sur le VIH et } \\
\text { la consommation de drogues) } \\
\text { - Manque de soutien adéquat } \\
\text { pour les personnes vivant avec le } \\
\text { VIH (25) }\end{array}$ \\
\hline Facteurs favorables & $\begin{array}{l}\text { - Avoir déjà été testé pour d'autres } \\
\text { ITSS (25) }\end{array}$ & $\begin{array}{l}\text { - Dépistage du VIH lancé/proposé } \\
\text { par les fournisseurs de soins de } \\
\text { santé (25) }\end{array}$ & $\begin{array}{l}\text { - Intégration du dépistage du VIH } \\
\text { aux services de santé courants (25) } \\
\text { - Offrir un large éventail d'options de } \\
\text { dépistage du VIH (25) }\end{array}$ \\
\hline
\end{tabular}

Provinces de l'Atlantique

\begin{tabular}{|c|c|c|c|}
\hline Obstacles & $\begin{array}{l}\text { - Difficulté à accéder à des services } \\
\text { de dépistage du VIH opportuns, } \\
\text { adaptés au genre et adaptés aux } \\
\text { jeunes (28) } \\
\text { - Manque d'accessibilité et de } \\
\text { confidentialité dans les petits } \\
\text { milieux communautaires (e.g. } \\
\text { relations personnelles entre la } \\
\text { famille et les professionnels de la } \\
\text { santé) }(28,62) \\
\text { - Perception d'un faible risque; } \\
\text { manque de connaissances sur le } \\
\text { VIH (62) }\end{array}$ & - s.o. & $\begin{array}{l}\text { - Manque de personnel et de } \\
\text { ressources pour la collaboration } \\
\text { entre les provinces de } \\
\text { l'Atlantique (62) } \\
\text { - Absence de politiques d'orientation } \\
\text { pour les programmes, ce qui } \\
\text { entraîne une discordance entre les } \\
\text { secteurs (28) }\end{array}$ \\
\hline Facteurs favorables & $\begin{array}{l}\text { - Dépistage du VIH chez les jeunes } \\
\text { dans des centres de santé sexuelle } \\
\text { spécialisés } \\
\text { - Accroître la sensibilisation, } \\
\text { l'éducation et l'information sur } \\
\text { le VIH; souligner l'importance de } \\
\text { la prévention, réduire les idées } \\
\text { fausses liées au VIH pour réduire la } \\
\text { stigmatisation }(28,62)\end{array}$ & $\begin{array}{l}\text { - Formation continue pour offrir des } \\
\text { services de consultation avant et } \\
\text { après le test et l'orientation vers les } \\
\text { services de santé appropriés après } \\
\text { le test (62) } \\
\text { - Accroître la sensibilisation, } \\
\text { l'éducation et l'information sur } \\
\text { le VIH; souligner l'importance de } \\
\text { la prévention, réduire les idées } \\
\text { fausses liées au VIH pour réduire la } \\
\text { stigmatisation }(28,62)\end{array}$ & $\begin{array}{l}\text { - Accès à des approches neutres et } \\
\text { adaptées au genre (services sans } \\
\text { stéréotypes ou iniquités fondés sur } \\
\text { le sexe) (28) } \\
\text { - Matériel éducatif et promotionnel } \\
\text { adapté aux jeunes (e.g. contenu } \\
\text { adapté à l'âge, mentorat par les } \\
\text { pairs, médias sociaux, programmes } \\
\text { téléphoniques et Internet, projets } \\
\text { artistiques) (62) } \\
\text { - Accroître la sensibilisation, } \\
\text { l'éducation et l'information sur } \\
\text { le VIH; souligner l'importance de } \\
\text { la prévention, réduire les idées } \\
\text { fausses liées au VIH pour réduire la } \\
\text { stigmatisation (28,62) } \\
\text { - Augmenter le nombre et les } \\
\text { types de sites de dépistage (e.g. } \\
\text { cliniques dans les écoles, sites de } \\
\text { dépistage mobiles) et les modalités } \\
\text { (e.g. points de service, dépistage } \\
\text { anonyme) (62) } \\
\text { Collaboration interorganisationnelle } \\
\text { et intersectorielle (28,62) } \\
\text { Participation des jeunes à } \\
\text { l'élaboration et à la mise en œuvre } \\
\text { d'initiatives de prévention du VIH } \\
\text { et du virus de l'hépatite C (28,62) }\end{array}$ \\
\hline
\end{tabular}


Tableau 3 : Caractéristiques sociodémographiques associées à l'augmentation du dépistage du VIH, obstacles au dépistage du VIH et facteurs le facilitant, par administration au Canada, 2009-2019 (suite)

\begin{tabular}{|c|c|c|c|}
\hline Province/territoire & Niveau de l'individu & $\begin{array}{l}\text { Niveau du fournisseur de } \\
\text { soins de santé }\end{array}$ & Niveau des politiques \\
\hline \multicolumn{4}{|c|}{ L'ensemble du Canada ou province/territoire non précisé } \\
\hline $\begin{array}{l}\text { Caractéristiques et comportements } \\
\text { sociodémographiques associés à un } \\
\text { dépistage accru du VIH }\end{array}$ & $\begin{array}{l}\text { - } \text { Plus jeune (54) } \\
\text { - Appartenir à une minorité } \\
\text { sexuelle (54) } \\
\text { - Sexe/genre féminin (54) } \\
\text { - Avoir des partenaires } \\
\text { occasionnels }(54,63) \\
\text { - Exposition potentielle due à la } \\
\text { consommation de drogues (63) }\end{array}$ & - s. o. & $\begin{array}{l}\text { - Prévalence élevée du VIH dans les } \\
\text { provinces et territoires (54) }\end{array}$ \\
\hline Obstacles & $\begin{array}{l}\text { - Anxiété et peur (en raison du long } \\
\text { délai entre le moment du test et } \\
\text { l'obtention des résultats, le fait } \\
\text { d'être jugé, peur de la maladie et } \\
\text { de la mort, violence familiale ou } \\
\text { communautaire) }(6,63) \\
\text { - Difficulté d'accès aux services de } \\
\text { santé et de dépistage (installations } \\
\text { médicales limitées) (6,63) } \\
\text { - Obstacles géographiques à l'accès } \\
\text { aux soins de santé (6) } \\
\text { - Difficulté d'accès aux services de } \\
\text { dépistage (63) } \\
\text { - Manque de confidentialité dans les } \\
\text { - Mervices de test (6) } \\
\text { - Manque de counseling avant et } \\
\text { - Manque de (6) } \\
\text { fournisseurs de soins de santé en } \\
\text { raison du contexte historique du } \\
\text { racisme, de la colonisation et de } \\
\text { l'homophobie (6,57) } \\
\text { - Perception du risque faible, } \\
\text { manque d'intérêt, sentiment de } \\
\text { santé }(6,63) \\
\text { - Stigmatisation liée au VIH } \\
\text { et criminalisation de la non- } \\
\text { divulgation du VIH (57) }\end{array}$ & $\begin{array}{l}\text { - Stigmatisation liée au VIH (57) } \\
\text { - Manque de confiance envers les } \\
\text { fournisseurs de soins de santé en } \\
\text { raison du contexte historique du } \\
\text { racisme, de la colonisation et de } \\
\text { l'homophobie (57) } \\
\text { - Perception de faible risque par les } \\
\text { fournisseurs de soins de santé (6) }\end{array}$ & $\begin{array}{l}\text { Stigmatisation liée au VIH } \\
\text { et criminalisation de la } \\
\text { non-divulgation du VIH (57) }\end{array}$ \\
\hline Facteurs favorables & $\begin{array}{l}\text { - Forte perception de connaissances } \\
\text { personnelles au sujet du VIH (54) } \\
\text { - Dépistage régulier du VIH (63) }\end{array}$ & $\begin{array}{l}\text { - Formation et sensibilisation des } \\
\text { fournisseurs de soins de santé (6) } \\
\text { - Fournisseurs de soins de santé } \\
\text { suggérant un test de dépistage du } \\
\text { VIH (63) } \\
\text { - Test oral autoadministré non } \\
\text { supervisé (48) }\end{array}$ & $\begin{array}{l}\text { - Tests anonymes (6) } \\
\text { - Intégration du dépistage du } \\
\text { VIH dans les soins médicaux } \\
\text { courants (63) } \\
\text { - } \text { Disponibilité de différentes } \\
\text { modalités de dépistage: } \\
\text { dépistage rapide (6), conseils } \\
\text { et dépistage volontaires du VIH } \\
\text { pour les couples (53), dépistage } \\
\text { du VIH sur Internet (33), test oral } \\
\text { autoadministré non supervisé (48) } \\
\text { - Améliorer la capacité des } \\
\text { fournisseurs de services de santé } \\
\text { (e.g. cliniques, organismes de } \\
\text { lutte contre le sida, organismes } \\
\text { communautaires) (6) } \\
\text { - Interventions et programmes } \\
\text { sexospécifiques (6) } \\
\text { - Accroître la sensibilisation au VIH } \\
\text { (e.g. au moyen de campagnes et } \\
\text { d'outils éducatifs) (6) }\end{array}$ \\
\hline
\end{tabular}

Abréviations : gbHARSAH, gais, bisexuels et autres hommes ayant des relations sexuelles avec des hommes; HARSAH, hommes ayant des relations sexuelles avec des hommes; ITSS, infections transmissibles sexuellement et par le sang; SIDA, syndrome d'immunodéficience acquise; s. o., sans objet; VIH, virus de l'immunodéficience humaine

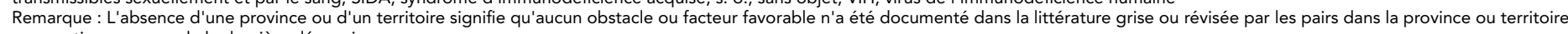
en question au cours de la dernière décennie

Bien que les provinces partagent de nombreux obstacles et facteurs favorables au dépistage du VIH, plusieurs tendances se sont dégagées dans des provinces particulières. Des études menées en Colombie-Britannique soulignent que la criminalisation et la stigmatisation du travail du sexe et les problèmes liés au statut d'immigrant constituent des obstacles majeurs au dépistage du VIH $(23,24,27)$. Les études menées en Ontario mettent davantage en évidence les obstacles culturels et les problèmes de stigmatisation et de peur des comportements associés au $\mathrm{VIH}$ que ceux dans autres régions $(38,60,64)$. Les 
études menées dans les provinces de l'Atlantique mettent en évidence de façon unique les services adaptés aux jeunes en tant que facteur favorable clé $(28,62)$. Les différences entre les provinces en matière d'obstacles et de facteurs favorables au dépistage du VIH étaient principalement attribuables à la présence des différentes populations clés et reflétaient les priorités régionales en matière de santé publique.

\section{Discussion}

Dans le présent examen systématique des études mixtes, nous avons inclus les résultats de 43 études menées au Canada pour documenter et comprendre les obstacles et facteurs favorables récents et émergents quant au dépistage du $\mathrm{VIH}$ au cours de la dernière décennie. La principale motivation de ce travail était d'orienter la recherche future et les mesures de santé publique vers l'atteinte de la première des trois cibles mondiales en matière de $\mathrm{VIH}$ au Canada, en tenant compte des populations clés et des contextes particuliers des provinces et territoires. Une autre motivation était de trouver des domaines d'intervention particuliers pour améliorer l'accès au dépistage du VIH dans un large éventail de contextes, notamment le dépistage accessible, peu coûteux et pratique, la confidentialité du dépistage, la réduction de la stigmatisation liée au $\mathrm{VIH}$, I'amélioration de l'éducation sur le VIH (e.g. modes de transmission, dépistage, traitements), la normalisation de l'offre de tests de dépistage du $\mathrm{VIH}$ et l'intégration du dépistage dans les pratiques de soins de santé de routine.

L'examen a fait ressortir des obstacles communs dans les populations clés et les provinces et territoires, notamment la perception d'un faible risque, la peur et la stigmatisation entourant le $\mathrm{VIH}$, le manque de connaissances sur le $\mathrm{VIH}$ et le dépistage, le manque de confidentialité pour les patients, l'accès limité à des services adaptés sur les plans culturel et linguistique et le manque de ressources pour le dépistage $(7,15)$. Cet examen a permis d'identifier plusieurs pratiques novatrices émergentes, notamment l'intégration du dépistage du $\mathrm{VIH}$ au point de service dans une variété de nouveaux contextes, y compris le dépistage du VIH sur Internet (33), les lieux de travail du sexe (27), les soins dentaires $(26,30)$, les salles d'urgence (52), les pharmacies (59) et les unités mobiles de dépistage $(26,27)$. Plusieurs méthodes de dépistage innovantes ont également été relevées, à savoir le conseil et le dépistage volontaires du VIH chez les couples (53), le prélèvement oral et le test oral autoadministré $(26,49)$ et les conseils après les tests offerts par les pairs (43). Les approches fondées sur le genre (28), la compétence des fournisseurs de soins de santé pour fournir des soins aux personnes queers et transgenres et les approches et interventions adaptées à ces dernières (50), le matériel d'éducation et de promotion adapté à l'âge, les sites de dépistage (e.g. cliniques scolaires pour les jeunes) et la mobilisation des jeunes dans l'élaboration et la mise en œuvre d'initiatives de prévention du VIH ont également été clairement identifiés comme des facteurs favorables importants (62).
Les données probantes résumées dans cet examen soulignent l'importance d'adapter les politiques et les programmes de santé publique aux contextes uniques de chaque province et territoire, notamment en ce qui concerne la répartition des populations clés et le fardeau de la maladie. Les stratégies potentielles pour améliorer l'accès au dépistage du VIH parmi les populations clés comprennent l'amélioration de l'accessibilité au dépistage du VIH en élargissant les options de dépistage disponibles et en assurant la promotion des initiatives de sensibilisation en matière de santé pour les populations difficiles à atteindre. Il est d'ailleurs essentiel d'assurer des services de santé inclusifs et non stigmatisants et d'intégrer les connaissances des membres de ces communautés pour améliorer l'acceptabilité du dépistage du VIH pour les populations clés. Les décideurs politiques et les fournisseurs de soins de santé devraient également tenir compte de l'intersectionnalité des identités et des expériences afin de mieux comprendre les facteurs particuliers du dépistage du VIH dans chaque population (65). Ces résultats soulignent l'importance d'adopter une approche centrée sur la personne pour le dépistage du VIH et la nécessité de joindre les personnes là où elles se trouvent.

Plusieurs obstacles et facteurs favorables relevés dans cet examen s'appliquent au niveau institutionnel et politique, ce qui indique peut-être un accent accru sur les déterminants en amont du dépistage du VIH au cours de la dernière décennie. Cette tendance récente souligne l'importance de l'action de santé publique au niveau systémique et suggère que les initiatives de dépistage du VIH pourraient être améliorées en tirant parti de l'expertise d'un éventail d'intervenants, notamment des partenaires communautaires, les soins de santé primaires, les services de réduction des méfaits et les autorités de la santé publique. L'augmentation du partenariat et de la collaboration intersectoriels pourrait offrir d'importantes possibilités de combler les lacunes en matière de dépistage et d'assurer un accès équitable au dépistage du $\mathrm{VIH}$.

Le Cadre d'action pancanadien reconnaît l'importance du dépistage pour atteindre les cibles mondiales en matière d'ITSS et présente des possibilités précises d'action qui correspondent aux facteurs favorables au dépistage relevés dans le présent examen (66). Comme l'indique le Plan d'action sur les infections transmissibles sexuellement et par le sang du gouvernement du Canada (67), I'amélioration de l'accès au dépistage des ITSS est un élément central d'une approche coordonnée visant à réduire I'incidence des ITSS au Canada, particulièrement lorsque les populations touchées de façon disproportionnée par les ITSS sont visées. Cet examen contribue aux connaissances existantes sur les facteurs de dépistage du VIH au Canada et met en lumière plusieurs lacunes et possibilités importantes qui peuvent éclairer les mesures de santé publique en vue d'atteindre cet objectif.

\section{Points forts et limites de l'étude}

L'un des principaux points forts de ce travail est la conception de l'examen systématique des études mixtes, qui synthétise 
les données quantitatives et qualitatives afin de répondre à des questions de recherche complexes comme l'identification des déterminants du dépistage du VIH (18). L'inclusion de multiples formes de données probantes crée une riche synthèse des obstacles et des facteurs favorables existants en combinant divers points de vue (i.e. données populationnelles et expériences individuelles) et produit des résultats qui sont directement pertinents pour les décideurs (22). De plus, la vaste portée permet d'identifier les obstacles et les facteurs favorables émergents et moins connus, ainsi que les tendances propres aux populations et aux provinces et territoires en matière de dépistage du VIH au Canada en vue d'éclairer les mesures de santé publique ciblées (68).

Néanmoins, cet examen comporte des limites. II est possible que certains travaux pertinents n'aient pas été retenus par notre stratégie de recherche; ainsi, certains obstacles ou facteurs favorables au dépistage pourraient être absents de cette synthèse. De plus, la nature des données a rendu impossible l'évaluation de la nature causale de l'un ou l'autre des obstacles ou facteurs favorables relevés.

Cet examen peut également être limité par un biais de publication, car la documentation publiée reflète les contextes et les priorités historiques et régionales, ce qui pourrait entraîner des lacunes dans la documentation relativement aux populations et contextes non prioritaires. Ainsi, bien que cet examen présente les résultats selon les populations et les provinces et territoires, certaines populations clés (e.g. les personnes qui utilisent des drogues par injection, les travailleurs du sexe, les immigrants, les communautés autochtones et les communautés africaines, antillaises et noires), certaines provinces (e.g. l'Alberta, le Manitoba et la Saskatchewan) et les territoires sont sousreprésentés, ce qui pourrait limiter la possibilité de généraliser à partir des résultats. De plus, des populations clés émergentes pourraient être absentes.

Enfin, la portée de cet examen était limitée aux obstacles et aux facteurs favorables au dépistage du VIH et pourrait donc entraîner l'omission d'autres obstacles et facteurs favorables communs et importants en matière de dépistage d'autres ITSS.

\section{Conclusion}

Le dépistage du VIH sert de porte d'entrée pour le traitement et la prévention du VIH et est un pilier central des efforts du Canada visant à réduire l'impact du VIH et d'autres ITSS sur la santé. Ce travail fournit une compréhension complète et détaillée des obstacles et des facteurs favorables au dépistage du VIH au Canada et souligne plusieurs facteurs importants qui peuvent être mis à profit pour accroître le dépistage du VIH. Les résultats fournissent des preuves clés pour influencer la pratique, les politiques et la recherche future en vue d'atteindre les cibles mondiales de lutte contre le $\mathrm{VIH}$.

\section{Déclaration des auteurs}

C. L. et C. B. F. ont également contribué à ces travaux : la conceptualisation, l'élaboration d'une stratégie de recherche, la sélection des travaux retenus pour inclusion, l'évaluation de la qualité, l'extraction des données, l'analyse et l'interprétation des données et la préparation des manuscrits.

\section{Intérêts concurrents}

Les auteurs n'ont aucun conflit d'intérêts à divulguer.

\section{Remerciements}

Nous tenons à remercier L. Pogany, J. Insogna et G. Tremblay du Centre de prévention et de contrôle des maladies infectieuses à l'Agence de la santé publique du Canada, à Ottawa, ainsi que A. Blair, L. Turcotte et D. Parisien du Bureau régional du Québec de l'Agence de la santé publique du Canada à Montréal pour leur contribution à la conceptualisation et à la conception du présent examen. Enfin, nous tenons à remercier K. Merucci et L. Glandon de la Bibliothèque de Santé Canada, à Ottawa, pour leur aide dans l'élaboration de la stratégie de recherche.

\section{Financement}

Ce travail a été appuyé par l'Agence de la santé publique du Canada.

\section{Références}

1. World Health Organization. Global HIV, hepatitis and STIs programme. HIV data and statistics. Geneva $(\mathrm{CH})$ : WHO; 2020 (accédé 2020-11-19). https://www.who.int/hiv/data/en/

2. Agence de la santé publique du Canada. Estimations de I'incidence et de la prévalence du $\mathrm{VIH}$, et des progrès réalisés par le Canada en ce qui concerne les cibles 90-90-90 pour le VIH. Ottawa (ON) : ASPC; 2018. https://www.canada. $\mathrm{ca} / \mathrm{fr} /$ sante-publique/services/publications/maladies-etaffections/esume-estimations-incidence-prevalence-vih-progr es-realises-canada-90-90-90.html

3. Samji H, Cescon A, Hogg RS, Modur SP, Althoff KN, Buchacz K, Burchell AN, Cohen M, Gebo KA, Gill MJ, Justice A, Kirk G, Klein MB, Korthuis PT, Martin J, Napravnik S, Rourke SB, Sterling TR, Silverberg MJ, Deeks S, Jacobson LP, Bosch RJ, Kitahata MM, Goedert JJ, Moore R, Gange SJ; North American AIDS Cohort Collaboration on Research and Design (NA-ACCORD) of leDEA. Closing the gap: increases in life expectancy among treated HIV-positive individuals in the United States and Canada. PLoS One 2013;8(12):e81355. DOI PubMed 
4. UNAIDS. Knowledge is power-Know your status, know your viral load. Geneva (CH): UNAIDS; 2018. https://www.unaids.org/en/resources/documents/2018/ knowledge-is-power-report

5. ONUSIDA. 90-90-90 Une cible ambitieuse de traitement pour aider à mettre fin à l'épidémie du sida. Genève $(\mathrm{CH})$ : ONUSIDA; 2014. https://www.unaids.org/sites/default/files/ media_asset/90-90-90_fr.pdf

6. Wertheimer S. Women and HIV testing in Canada: barriers and recommendations as identified by service providers: a summary of key research findings. Ottawa (ON): Canadian AIDS Society; 2011. https://www.conaids.ca/wp-content/ uploads/Women-and-HIV-Testing-in-Canada-A-Summ ary-of-Key-Research-Findings.pdf

7. Traversy GP, Austin T, Ha S, Timmerman K, Gale-Rowe $M$. Un aperçu des récentes données probantes sur les obstacles et les facteurs favorables au dépistage du virus de l'immunodéficience humaine. Relevé des maladies transmissibles au Canada 2015;41(12):445-63. DOI

8. MacCarthy S, Poteat T, Xia Z, Roque NL, Hyun Jin Kim A, Baral S, Reisner SL. Current research gaps: a global systematic review of HIV and sexually transmissible infections among transgender populations. Sex Health 2017;14(5):456-68. DOl PubMed

9. Degenhardt L, Charlson F, Stanaway J, Larney S, Alexander LT, Hickman M, Cowie B, Hall WD, Strang J, Whiteford $\mathrm{H}$, Vos T. Estimating the burden of disease attributable to injecting drug use as a risk factor for HIV, hepatitis C, and hepatitis B: findings from the Global Burden of Disease Study 2013. Lancet Infect Dis 2016;16(12):1385-98. DOI PubMed

10. Baral S, Beyrer C, Muessig K, Poteat T, Wirtz AL, Decker MR, Sherman SG, Kerrigan D. Burden of HIV among female sex workers in low-income and middle-income countries: a systematic review and meta-analysis. Lancet Infect Dis 2012;12(7):538-49. DOI PubMed

11. Iles $\mathrm{L}$, Consolacion T, Wong J, Grennan T, Gilbert M, Prescott C, Moore D. HIV diagnoses and testing patterns among young gay, bisexual and other men who have sex with men: an analysis of HIV surveillance data in British Columbia, 2008-2015. Can J Public Health 2019;110(5):668-74. DOl PubMed

12. Dolan $K$, Wirtz AL, Moazen B, Ndeffo-Mbah M, Galvani A, Kinner SA, Courtney R, McKee M, Amon JJ, Maher L, Hellard M, Beyrer C, Altice FL. Global burden of HIV, viral hepatitis, and tuberculosis in prisoners and detainees. Lancet 2016;388(10049):1089-102. DOI PubMed

13. Fitzgerald-Husek A, Van Wert MJ, Ewing WF, Grosso AL, Holland CE, Katterl R, Rosman L, Agarwal A, Baral SD. Measuring stigma affecting sex workers (SW) and men who have sex with men (MSM): A systematic review. PLoS One 2017;12(11):e0188393. DOI PubMed

14. DeBeck K, Cheng T, Montaner JS, Beyrer C, Elliott R, Sherman S, Wood E, Baral S. HIV and the criminalisation of drug use among people who inject drugs: a systematic review. Lancet HIV 2017;4(8):e357-74. DOI PubMed
15. Kaai S, Bullock S, Burchell AN, Major C. Factors that affect HIV testing and counseling services among heterosexuals in Canada and the United Kingdom: an integrated review. Patient Educ Couns 2012;88(1):4-15. DOI PubMed

16. Bolsewicz K, Vallely A, Debattista J, Whittaker A, Fitzgerald L. Factors impacting HIV testing: a review-perspectives from Australia, Canada, and the UK. AIDS Care 2015;27(5):570-80. DOI PubMed

17. Deblonde J, De Koker P, Hamers FF, Fontaine J, Luchters $\mathrm{S}$, Temmerman M. Barriers to HIV testing in Europe: a systematic review. Eur J Public Health 2010;20(4):422-32. DOl PubMed

18. Pluye $P$, Hong QN. Combining the power of stories and the power of numbers: mixed methods research and mixed studies reviews. Annu Rev Public Health 2014;35:29-45. DOl PubMed

19. Moher D, Liberati A, Tetzlaff J, Altman DG; PRISMA Group. Preferred reporting items for systematic reviews and meta-analyses: the PRISMA statement. BMJ 2009;339:b2535. DOI PubMed

20. Hong QN, Gonzalez-Reyes A, Pluye P. Improving the usefulness of a tool for appraising the quality of qualitative, quantitative and mixed methods studies, the Mixed Methods Appraisal Tool (MMAT). J Eval Clin Pract 2018;24(3):459-67. DOI PubMed

21. Pace R, Pluye P, Bartlett G, Macaulay AC, Salsberg J, Jagosh J, Seller R. Testing the reliability and efficiency of the pilot Mixed Methods Appraisal Tool (MMAT) for systematic mixed studies review. Int J Nurs Stud 2012;49(1):47-53. DOl PubMed

22. Hong $\mathrm{QN}$, Pluye $\mathrm{P}$, Bujold $\mathrm{M}$, Wassef $\mathrm{M}$. Convergent and sequential synthesis designs: implications for conducting and reporting systematic reviews of qualitative and quantitative evidence. Syst Rev 2017;6(1):61. DOI PubMed

23. Anderson S, Shannon K, Li J, Lee Y, Chettiar J, Goldenberg S, Krüsi A. Condoms and sexual health education as evidence: impact of criminalization of in-call venues and managers on migrant sex workers access to HIV/STI prevention in a Canadian setting. BMC Int Health Hum Rights 2016;16(1):30. DOI PubMed

24. Armstrong $\mathrm{HL}$, Wang L, Zhu J, Lachowsky NJ, Card KG, Wong J, Jollimore J, Edward J, Roth EA, Hogg RS, Moore DM. HIV testing among a representative community sample of gay, bisexual, and other men who have sex with men in Vancouver, Canada. AIDS Behav 2019;23(2):347-58. DOI PubMed

25. Boyd SE, Allison J, Penney CC, Burt K, Allison D, Daley PK. Timeliness of diagnosis of HIV in Newfoundland and Labrador, Canada: a mixed-methods study. JAMMI 2019;4(1):15-23. DOI

26. Brondani M, Chang S, Donnelly L. Assessing patients' attitudes to opt-out HIV rapid screening in community dental clinics: a cross-sectional Canadian experience. BMC Res Notes 2016;9:264. DOI PubMed 
27. Deering KN, Montaner JS, Chettiar J, Jia J, Ogilvie G, Buchner C, Feng C, Strathdee SA, Shannon K. Successes and gaps in uptake of regular, voluntary HIV testing for hidden street- and off-street sex workers in Vancouver, Canada. AIDS Care 2015;27(4):499-506. DOI PubMed

28. Dube A, Harris G, Gahagan J, Doucet S. Bridging the silos in HIV and Hepatitis $C$ prevention: a cross-provincial qualitative study. Int J Public Health 2017;62(7):739-46. DOI PubMed

29. Engler K, Rollet K, Lessard D, Thomas R, Lebouché B. Explaining the presence of "heterosexual" female clients of a rapid HIV testing site located in the gay village of Montreal, Quebec. J Prim Care Community Health 2016;7(2):122-9. DOI PubMed

30. Feng I, Brondani M, Chong KL, Donnelly L. Evaluating point-of-care HIV screening in dental hygiene education settings: patient, faculty, and student perspectives. J Dent Educ 2018;82(8):819-27. DOI PubMed

31. Gahagan JC, Fuller JL, Proctor-Simms EM, Hatchette TF, Baxter LN. Barriers to gender-equitable HIV testing: going beyond routine screening for pregnant women in Nova Scotia, Canada. Int J Equity Health 2011;10(1):18. DOI PubMed

32. Gilbert M, Cook D, Steinberg M, Kwag M, Robert W, Doupe G, Krajden M, Rekart M. Targeting screening and social marketing to increase detection of acute HIV infection in men who have sex with men in Vancouver, British Columbia. AIDS 2013;27(16):2649-54. DOI PubMed

33. Gilbert M, Hottes TS, Kerr T, Taylor D, Fairley CK, Lester R, Wong T, Trussler T, Marchand R, Shoveller J, Ogilvie G. Factors associated with intention to use internet-based testing for sexually transmitted infections among men who have sex with men. J Med Internet Res 2013;15(11):e254. DOI PubMed

34. Holtzman S, Landis L, Walsh Z, Puterman E, Roberts D, Saya-Moore K. Predictors of HIV testing among men who have sex with men: a focus on men living outside major urban centres in Canada. AIDS Care 2016;28(6):705-11. DOI PubMed

35. Iqbal S, De Souza LR, Yudin MH. Acceptability, predictors and attitudes of Canadian women in labour toward point-ofcare HIV testing at a single labour and delivery unit. Can J Infect Dis Med Microbiol 2014;25(4):201-6. DOI PubMed

36. Kesler MA, Kaul R, Loutfy M, Myers T, Brunetta J, Remis RS, Gesink D. Prosecution of non-disclosure of HIV status: potential impact on HIV testing and transmission among HIV-negative men who have sex with men. PLoS One 2018;13(2):e0193269. DOI PubMed

37. Knight R, Small W, Shoveller J. How do 'public' values influence individual health behaviour? An empirical-normative analysis of young men's discourse regarding HIV testing practices. Public Health Ethics 2016;9(3):264-75. DOl PubMed

38. Knight R, Small W, Shoveller JA. HIV stigma and the experiences of young men with voluntary and routine HIV testing. Sociol Health Illn 2016;38(1):153-67. DOI PubMed
39. Lau L, Wudel B, Lee E, Darraj M, Richert $Q$, Trajtman A, Bresler K, Bullard J, Kasper K, Becker M, Keynan Y. Evaluation of the utility of point-of-care HIV testing on a Canadian internal medicine inpatient unit. Can J Infect Dis Med Microbiol 2017;2017:8495307. DOI PubMed

40. Lazarus L, Patel S, Shaw A, Leblanc S, Lalonde C, Hladio M, Mandryk K, Horvath C, Petrcich W, Kendall C, Tyndall MW; Proud Community Advisory Committee. Uptake of community-based peer administered HIV point-of-care testing: findings from the PROUD study. PLoS One 2016;11(12):e0166942. DOI PubMed

41. Lessard D, Lebouché B, Engler K, Thomas R, Machouf N. Explaining the appeal for immigrant men who have sex with men of a community-based rapid HIV-testing site in Montreal (Actuel sur Rue). AIDS Care 2015;27(9):1098-103. DOI PubMed

42. Lewis NM, Gahagan JC, Stein C. Preferences for rapid point-of-care HIV testing in Nova Scotia, Canada. Sex Health 2013;10(2):124-32. DOI PubMed

43. Markwick N, Ti L, Callon C, Feng C, Wood E, Kerr T. Willingness to engage in peer-delivered HIV voluntary counselling and testing among people who inject drugs in a Canadian setting. J Epidemiol Community Health 2014;68(7):675-8. DOI PubMed

44. O'Byrne P, Bryan A. Anonymous HIV testing and public health in Ontario, Canada: understanding HIV surveillance. Surveill Soc 2013;11(1):35-54. DOI

45. O'Byrne P, Bryan A, Woodyatt C. Nondisclosure prosecutions and HIV prevention: results from an Ottawa-based gay men's sex survey. J Assoc Nurses AIDS Care 2013;24(1):81-7. DOI PubMed

46. O'Byrne P, Watts J. Include, differentiate and manage: gay male youth, stigma and healthcare utilization. Nurs Inq 2014 Mar;21(1):20-9. DOI PubMed

47. O'Byrne $P$, Willmore J, Bryan A, Friedman DS, Hendriks A, Horvath C, Massenat D, Bouchard C, Remis RS, Etches V. Nondisclosure prosecutions and population health outcomes: examining HIV testing, HIV diagnoses, and the attitudes of men who have sex with men following nondisclosure prosecution media releases in Ottawa, Canada. BMC Public Health 2013;13(1):94. DOI PubMed

48. Pai NP, Smallwood M, Gulati D, Lapczak N, Musten A, Gaydos C, Johnston C, Steben M, Wong T, Engel N, Kim J. What do key stakeholders think about HIV self-testing in Canada? Results from a cross-sectional survey. AIDS Behav 2018;22(2):606-15. DOI PubMed

49. Pai NP, Bhargava M, Joseph L, Sharma J, Pillay S, Balram B, Tellier PP. Will an unsupervised self-testing strategy be feasible to operationalize in Canada? Results from a pilot study in students of a large canadian university. Aids Res Treat 2014;2014(747619):1-8. DOI PubMed 
50. Rich A, Scott K, Johnston C, Blackwell E, Lachowsky N, Cui Z, Sereda P, Moore D, Hogg R, Roth E. Sexual HIV risk among gay, bisexual and queer transgender men: findings from interviews in Vancouver, Canada. Cult Health Sex 2017;19(11):1197-209. DOI PubMed

51. Scheim Al, Travers R. Barriers and facilitators to HIV and sexually transmitted infections testing for gay, bisexual, and other transgender men who have sex with men. AIDS Care 2017;29(8):990-5. DOI PubMed

52. Stenstrom R, Ling D, Grafstein E, Barrios R, Sherlock C, Gustafson R, Osati F, Poureslami I, Anis A. Prevalence of HIV infection and acceptability of point-of-care testing in a Canadian inner-city emergency department. Can J Public Health 2016 Oct;107(3):e291-5. DOl PubMed

53. Stephenson R, Chard A, Finneran C, Sullivan P. Willingness to use couples voluntary counseling and testing services among men who have sex with men in seven countries. AIDS Care 2014;26(2):191-8. DOI PubMed

54. Worthington CA, Calzavara LM, White SJ, Allman D, Tyndall MW. Individual and jurisdictional factors associated with voluntary HIV testing in Canada: results of a national survey, 2011. Can J Public Health 2014;106(2):e4-9. DOI PubMed

55. Worthington CA, O'Brien KK, Mill J, Caine V, Solomon $P$, Chaw-Kant J. A mixed-methods outcome evaluation of a mentorship intervention for Canadian nurses in HIV care. J Assoc Nurses AIDS Care 2016;27(5):677-97. DOI PubMed

56. Barbour TL. HIV testing in Nova Scotia: an Indigenous perspective on access and acceptability. Halifax, (NS): Dalhousie University; 2017. https://dalspace.

library.dal.ca/bitstream/handle/10222/73293/

Barbour-Tammy-MA-August-29-2017.

pdf?sequence $=3$ \&isAllowed $=y$

57. CATIE. Dialogue délibératif pour joindre les personnes non diagnostiquées pour le VIH : mettre à l'échelle les approches de l'élaboration des programmes d dépistage du VIH et de l'arrimage à la prévention et aux soins. Jeudi 13 octobre et vendredi 14 octobre 2016. Rapport de la réunion. Toronto (ON) : La source canadienne de renseignements sur le VIH et l'hépatite C; 2016. https://www.catie.ca/ga-pdf. php?file=sites/default/files/Reaching-the-hiv-undiagnosedFR.pdf

58. Centre SIDA Amitié. Rapport de recommandations de lutte contre le VIH et le sida pour atteindre les cibles mondiales à l'échelle régionale. Saint-Jérôme ( $\mathrm{QC})$ : Centre SIDA Amitié; 2019. https://centresidaamitie.app.box.com/s/2bq649o1iue olzfpzzqqydz7oc9t419n

59. Gahagan J, Stein C, Campbell A. Report on anonymous HIV Testing program and perceptions of acceptability of rapid point-of-care testing at Halifax Sexual Health Centre, Halifax, Nova Scotia. Halifax (NS): Halifax Sexual Health Center; 2012. https://novascotia.ca/aids/documents/ Report-Anonymous-HIV-Testing-Program-HSHC-2012.pdf

60. Konkor I. HIV vulnerabilities among heterosexual African, Caribbean and other Black men in London, Ontario. London (ON): University of Western Ontario; 2019. https://ir.lib.uwo. $\mathrm{ca} / \mathrm{etd} / 6243 /$
61. Messier-Peet M, Apelian H, Moodie E, Cox J, Hart T, Grace D, Moore D, Lachowsky N, Jollimore J, Rodrigues R, Sparling D, Noor S, Olarewaju G, Armstrong H, Lambert G. Investigating factors associated with sub-optimal HIV testing among high-risk gay, bisexual, and other men who have sex with men: results from Engage Montreal 2017-2018. Canadian Association of HIV Researchers Conference; 2019 May 10-12; Saskatoon, SK. https://www.engage-men.ca/ our-work/posters/

62. Gender and Health Promotion Studies Unit. Our Youth, Our Response: Building capacity for effective HIV/HCV policy and programming responses across the Atlantic Region. Final report. Halifax (NS): Dalhousie University; 2014. https://www.dal.ca/diff/gahps/research-projects/oyor.html

63. Agence de la santé publique du Canada. I-Track: surveillance améliorée du $\mathrm{VIH}$ et de l'hépatite $\mathrm{C}$ ainsi que des comportements à risque chez les utilisateurs de drogues injectables au Canada - Rapport sur la phase 3 (2010-2012). Ottawa (ON) : Gouvernement du Canada; 2018. https:// www.canada.ca/fr/sante-publique/services/publications/ maladies-et-affections/itrack-surveillance-amelioree-vih-he patite-comportements-risque-utilisateurs-drogu es-injectables-canada-phase-3.html

64. Vannice S. Barriers, access, resources, and knowledge (B.A.R.K.): an analysis of HIV testing vis Women's Voices in Ottawa: Ottawa (ON): University of Ottawa; 2016. https://ruor.uottawa.ca/bitstream/10393/35602/5/Vannice_ Sarah_2016_thesis.pdf

65. Heard E, Fitzgerald L, Wigginton B, Mutch A. Applying intersectionality theory in health promotion research and practice. Health Promot Int 2020;35(4):866-76. DOI PubMed

66. Agence de la santé publique du Canada. Réduction des répercussions sur la santé des infections transmissibles sexuellement et par le sang au Canada d'ici 2030 : un cadre d'action pancanadien sur les ITSS. Ottawa (ON) : Gouvernement du Canada; 2018. https://www.canada. $\mathrm{ca} / \mathrm{fr} /$ sante-publique/services/maladies-infectieuses/ sante-sexuelle-infections-transmissibles-sexuellement/ rapports-publications/infections-transmissible s-sexuellement-sang-cadre-action.html

67. Agence de la santé publique du Canada. Accélérer notre intervention : plan d'action quinquennal du gouvernement du Canada sur les infections transmissibles sexuellement et par le sang. Ottawa (ON) : Gouvernement du Canada; 2019. https://www.canada.ca/fr/sante-publique/services/ rapports-publications/accelerer-notre-intervention -plan-action-quinquennal-infections-transmissibles -sexuellement-sang.html

68. Cerigo H, Quesnel-Vallée A. Systematic mixed studies reviews: leveraging the literature to answer complex questions through the integration of quantitative and qualitative evidence. Int J Public Health 2020;65(5):699-703. DOI PubMed 